\title{
Modeling Random Forwarding Actions for Information Diffusion over Mobile Social Networks
}

\author{
Ling Li, ${ }^{1,2}$ Min Liu, ${ }^{1}$ Weiming Shen, ${ }^{1,3}$ and Guoqing Cheng ${ }^{2}$ \\ ${ }^{1}$ School of Electronic and Information Engineering, Tongji University, Shanghai 201804, China \\ ${ }^{2}$ School of Information Engineering, Jingdezhen Ceramic Institute, Jingdezhen 333403, China \\ ${ }^{3}$ The Key Laboratory of Embedded System and Service Computing, Ministry of Education, Tongji University, Shanghai 200092, China
}

Correspondence should be addressed to Min Liu; lmin@tongji.edu.cn

Received 4 February 2016; Revised 23 May 2016; Accepted 30 May 2016

Academic Editor: Hua Lu

Copyright (c) 2016 Ling Li et al. This is an open access article distributed under the Creative Commons Attribution License, which permits unrestricted use, distribution, and reproduction in any medium, provided the original work is properly cited.

\begin{abstract}
Modeling information diffusion over social networks has attracted a lot of attention from both academia and industry. Based on universal generating function method and discrete stress-strength interference theory, a novel method is proposed to model the users' random forwarding actions, and the most susceptible users are extracted. The effect of a user on information diffusion is quantified as node susceptibility (NS), and NS is defined as the probability that quantity of information (message) the user forwards is larger than that he receives. The model can address three questions: which users are most susceptible, which types of information they are most susceptible to, and when they are most susceptible. The solutions of these questions are very helpful for the practitioners. A case study is used to illustrate the feasibility and practicality of the proposed model.
\end{abstract}

\section{Introduction}

With the development of Internet and mobile information technologies, social networks have incrementally become the most popular applications $[1,2]$. A mobile social network connects organizations or individuals using smartphones with sharing of information through social networking applications such as MySpace, Facebook, and scientific collaboration networks [3,4]. Mobile social networks are present in virtual communities and work with web-based social networks to spread content, increase accessibility, and connect users from wherever they are [5]. One of the distinguishing factors of social networks is the ability to enable people to simultaneously share information with large number of peers [6]. Due to its diverse implication, research on social networking from various perspectives has received remarkable attention. In the area of social networks, modeling and analyzing their networking structures [7], dynamic evolution [8], and the characteristics of information diffusion [9] are hot topics.

More recently, the smartphones have increased rapidly, and social networks are experiencing explosive growth, not only in the number of communities but also in the overall population [10]. For example, Facebook has over 1.18 billion monthly active users as of August 2015 [11]. Everyday users are sharing and exchanging the information by means of "word-of-mouth" communications in such large-scale social networks. Under such circumstances, in-depth analyzing and quantifying random actions (to forward the information or not) of users for information diffusion over social networks have become more and more important [12]. For each user (a human in a social network or a website on the Internet), whether to forward the information depends on several uncertain factors, including the interestingness of the information, the reliability of the information, egoistic motivation, and altruistic motivation [13]. Whatever the motivation, forwarding actions indicate that the user is susceptible to the information, and the higher the frequency, the more susceptible the user. In this sense, the study of random forwarding actions can help the politicians/enterprises to identify the susceptible users and then to achieve the most effective advocation/advertisement. On the other hand, the global diffusion of detrimental information (e.g., computer viruses, rumors) causes great damage to society. It is of great importance to identify the susceptible users and timely quarantine them [14]. Therefore, from the point of view of the 
security, the study of random forwarding actions can help to prevent the diffusion of detrimental information.

The rapid developments of communication and information technologies enable us to access, collect, and store the real-world big data on information diffusion, making the related research meaningful and versatile and, meanwhile, more challenging. Back to 2001, Pastor-Satorras and Vespignani [15] studied epidemic/computer virus spreading over network, indicating that the study on information diffusion originates from the study on epidemic/computer virus diffusion. One of the earliest and prominent studies on information diffusion is [16], which studied and analyzed the dynamics of information dissemination through blogspace from two points of view: macroscopic and microscopic. Subsequently, there are numerous related works addressing information diffusion over social networks. Here, we summarize the most representative works that are relevant to our study. The existing related works can be broadly classified into two categories. The first one focuses on analyzing the effect of network structure on information diffusion [1721]. In [17], the authors studied the scaling law of a few large networks and showed that the vertex connectivity obeys a scale-free distribution of power law. Donetti et al. [18] reported that scale-free structures may be generated by optimal designing for network mechanisms. The work [19] reported that the scale-free network can optimize the network performance. Recently, how the network structure of microblog influences information diffusion was studied in [20]. By studying followers' topology, the authors presented an invariant characteristic that the users' followers count obeys a distribution of power law with exponent near 2 . In [21], the authors comparatively explored the network structure, geographic distribution of users, and interaction pattern in social networks. Based on the study, the authors suggested that information can be organized by a few central users bridging small communities.

The second category focuses on analyzing the effect of network nodes (users) on information diffusion using different mathematical models [22-28]. Kimura et al. [22] considered the optimization problem of extracting the most influential nodes over a social network. Later, Yu et al. [23] proposed a community-based greedy algorithm to mine top- $k$ influential nodes over mobile social networks. By identifying the important information nodes, Ilyas et al. [24] studied how to restrain the private information diffusion. As social networks (e.g., Twitter and Facebook) become ubiquitous, the global effect of a node on diffusion rate on Twitter was studied in $[25,26]$. More recently, Saito et al. [27] proposed an efficient method to find a new kind of influential nodes (supermediators) over a social network and characterized the properties of supermediators. From another perspective, Belák et al. [28] studied the effect of hidden nodes on information diffusion and characterized information cascades.

The study presented in this paper focuses on modeling nodes' random forwarding actions and analyzing the effect of these actions on information diffusion; that is, our study falls into the second category. Although the above-cited literatures provide systematic approaches and useful tools for analyzing the effect of network nodes on information diffusion, the majority of them neglect the dynamic and random characteristics of this problem. Given the complexity and uncertainty of social network, it is difficult for nodes to maintain the same effect on information diffusion during different periods. In addition, most of them mainly focus on extracting the most influential nodes, while mining the most susceptible nodes has not been well investigated. In fact, quantifying nodes' random forwarding actions and finding the most susceptible nodes also play an important role for information diffusion. On the one hand, from the academic research point of view, for information to diffuse, it in essence relies on nodes to forward the information that they receive. However, whether to forward the information is uncertain and depends on many factors. Therefore, quantifying nodes' random forwarding actions can help to objectively and rationally analyze which nodes are susceptible to the information and when they are most susceptible. On the other hand, from the practical significance point of view, for politicians/enterprises, it is easier to obtain advocation/purchasing from the susceptible nodes, rather than the influential nodes. Therefore, identifying the most susceptible nodes can help politicians/enterprises to achieve the most effective advocation/advertisement.

This work is motivated by the challenges of quantifying nodes' random forwarding actions and finding the most susceptible nodes, at the same time emphasizing the dynamics characteristics of this problem. The study aims to address three key questions: (1) which nodes are most susceptible. (2) which types of information they are most susceptible to. and (3) when they are most susceptible. To this end, a novel and efficient model for analyzing the effect of nodes on information diffusion is proposed based on universal generating function (UGF) method and discrete stress-strength interference (DSSI) theory. Stress-strength interference models have been widely used in component reliability analysis, but to the best of our knowledge, it is the first time that stress-strength interference model is applied to information diffusion analysis. In our model, the effect of node is quantified as node susceptibility (NS), which is relevant to two random variables: quantity of information (message) that the node receives and quantity of information (message) that the node forwards, and NS is defined as the probability that the latter (strength) is larger than the former (stress). Based on NS, the proposed model can help decisionmakers to dynamically identify which nodes are most susceptible to the corresponding information at different periods of time. The innovations and practical significance of this paper are as follows.

(i) Approach Innovations. To model random forwarding actions over mobile social networks, DSSI model is applied to information diffusion analysis for the first time. Unlike the continuous stress-strength interference (CSSI) model, DSSI model can calculate system reliability (NS in this paper) based on observations of stress and strength when the distributions of stress and strength are unavailable. Moreover, since the stress and strength in the paper are discrete random variables, UGF method is utilized to represent their probability mass functions for the calculation of NS. In this sense, 
the calculation of NS is based on actual observation data, rather than being dependent on decision-makers' subjective judgments; therefore, the decision results are objective and will be updated with the updated observation data.

(ii) Practical Significance. For the decision-makers (practitioners), modeling and decision process are easy to implement, since the calculation of NS is based on the observations of random variables which can be obtained directly from the database. In conventional approaches, decision-makers need to know specialized knowledge of filtering appropriate criteria from a lot of criteria and specifying the weights of criteria for optimization decisions, but, here, they only need to record the observations of the relevant variables, which can simplify the process of decision.

The rest of this paper is organized as follows. Section 2 describes the theoretical background of the proposed model. Model formulation is presented in Section 3. In Section 4, a case study is presented to illustrate the feasibility and efficiency of the proposed model. The paper ends with conclusions in Section 5.

\section{Theoretical Background}

Before describing the mathematical model, we introduce some definitions and notations related to universal generating function (UGF) method and discrete stress-strength interference (DSSI) model. They will be used in Section 3.

2.1. Brief Description of UGF Method. We put emphasis on the basic concept but not the fundamental mathematics of UGF method. Ushakov [29] first introduced the concept of UGF. Then, Lisnianski and Levitin [30] and Levitin [31] applied UGF method to reliability analysis and optimization of multistate system.

Suppose that a discrete random variable (r.v.) $X$ has a probability mass function (p.m.f.) characterized by the vector $x$ consisting of the possible values of $X$ and the vector $p$ consisting of the corresponding probabilities, which can be formulated by the following expressions: $x=\left(x_{1}, x_{2}, \ldots, x_{k}\right)$ and $p=\left(p_{1}, p_{2}, \ldots, p_{k}\right)$, where $p_{i}=\operatorname{Pr}\left(X=x_{i}\right), i=$ $1,2, \ldots, k$.

Definition 1 (UGF of discrete random variable). The UGF of $X$ is defined as a polynomial function of variable $z, u_{X}(z)$, and

$$
u_{X}(z)=p_{1} z^{x_{1}}+p_{2} z^{x_{2}}+\cdots+p_{k} z^{x_{k}}=\sum_{i=1}^{k} p_{i} z^{x_{i}}
$$

It should be mentioned that there exists a one-to-one correspondence between the p.m.f. and UGF of a discrete r.v. This means that, for an arbitrary discrete r.v., its UGF is uniquely determined by its p.m.f..

Definition 2 (UGF of discrete random variables). Consider $n$ independent discrete r.v. $X_{1}, X_{2}, \ldots, X_{n}$. Let the UGF of each r.v. be $u_{X_{1}}(z), u_{X_{2}}(z), \ldots, u_{X_{n}}(z)$, respectively, and $f\left(X_{1}, X_{2}, \ldots, X_{n}\right)$ an arbitrary function of variables
$X_{1}, X_{2}, \ldots, X_{n}$. Then, by employing composition operator $\otimes$, the UGF of $f\left(X_{1}, X_{2}, \ldots, X_{n}\right), u_{f}(z)$ can be obtained as follows:

$$
u_{f}(z)=\bigotimes\left(u_{X_{1}}(z), u_{X_{2}}(z), \ldots, u_{X_{n}}(z)\right)
$$

Definition 3 (composition operator $\bigotimes$ ). According to Definition $1, u_{X_{i}}(z)=\sum_{j_{i}=1}^{k_{i}} p_{i j_{i}} z^{x_{i j_{i}}}, i=1,2, \ldots, n$, where $k_{1}, k_{2}, \ldots$, $k_{n}$ are, respectively, the number of possible values of each r.v. To obtain the UGF of $f\left(X_{1}, X_{2}, \ldots, X_{n}\right)$, composition operator $\otimes$ is defined as follows:

$$
\begin{gathered}
\bigotimes\left(\sum_{j_{1}=1}^{k_{1}} p_{1 j_{1}} z^{x_{1 j_{1}}}, \sum_{j_{2}=1}^{k_{2}} p_{2 j_{2}} z^{x_{2 j_{2}}}, \ldots, \sum_{j_{n}=1}^{k_{n}} p_{n j_{n}} z^{x_{n j_{n}}}\right) \\
=\sum_{j_{1}=1}^{k_{1}} \sum_{j_{2}=1}^{k_{2}} \cdots \sum_{j_{n}=1}^{k_{n}}\left(\prod_{i=1}^{n} p_{i j_{i}} z^{f\left(x_{1 j_{1}}, x_{2 j_{2}}, \ldots, x_{n j_{n}}\right)}\right) .
\end{gathered}
$$

Property 1. In the operation of UGF, commutative law and associative law are applicable:

$$
\begin{aligned}
& u_{f}(z)=\bigotimes\left(u_{X_{1}}(z), u_{X_{2}}(z), \ldots, u_{X_{i}}(z), u_{X_{i+1}}(z), \ldots,\right. \\
& \left.u_{X_{n}}(z)\right)=\bigotimes\left(u_{X_{1}}(z), u_{X_{2}}(z), \ldots, u_{X_{i+1}}(z), u_{X_{i}}(z),\right. \\
& \left.\ldots, u_{X_{n}}(z)\right), \\
& u_{f}(z)=\bigotimes\left(u_{X_{1}}(z), u_{X_{2}}(z), \ldots, u_{X_{i}}(z), u_{X_{i+1}}(z), \ldots,\right. \\
& \left.u_{X_{n}}(z)\right) \\
& =\bigotimes\left(\bigotimes\left(u_{X_{1}}(z), u_{X_{2}}(z), \ldots, u_{X_{i+1}}(z)\right),\right. \\
& \left.\bigotimes\left(u_{X_{i}}(z), \ldots, u_{X_{n}}(z)\right)\right) .
\end{aligned}
$$

2.2. Discrete Stress-Strength Interference (DSSI) Model. Stress-strength interference model [32] has been widely used for reliability analysis of component, where "component" is not necessarily the raw goods or parts but can be an entire system. Stress-strength analysis is an efficient tool used in reliability engineering.

Definition 4 (component reliability). Let $S_{1}$ and $S_{2}$ denote stress on a component and strength of a component, respectively; then, the component reliability denoted by $R$ is defined as

$$
R=\operatorname{Pr}\left(S_{2}>S_{1}\right) .
$$

Equation (5) is the most basic expression of the stressstrength interference model, which indicates that the component reliability is defined as the probability that the strength is larger than the stress.

If $S_{1}$ and $S_{2}$ are treated as continuous r.v. and their probability density functions are denoted by $f_{1}\left(S_{1}\right)$ and $f_{2}\left(S_{2}\right)$, respectively, (5) can be rewritten as

$$
R=\int_{-\infty}^{+\infty} f_{1}\left(S_{1}\right) \cdot\left[\int_{S_{1}}^{+\infty} f_{2}\left(S_{2}\right) d S_{2}\right] d S_{1}
$$




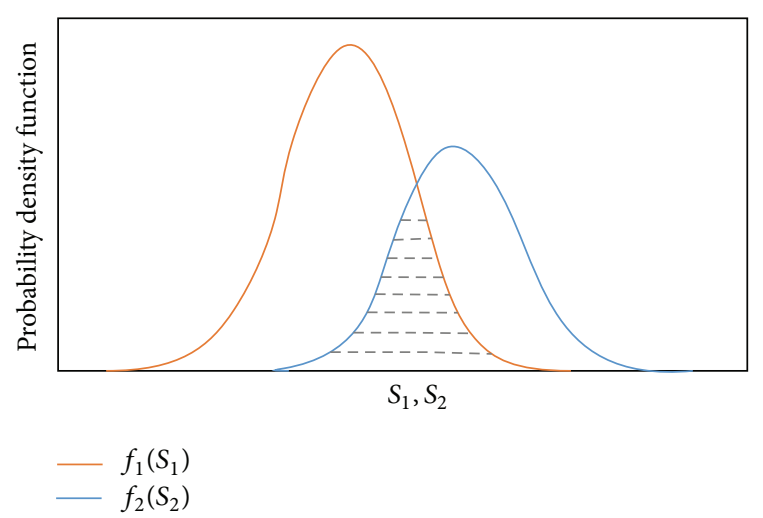

FIGURE 1: Component reliability as overlap of stress and strength.

or

$$
R=\int_{-\infty}^{+\infty} f_{2}\left(S_{2}\right) \cdot\left[\int_{-\infty}^{S_{2}} f_{1}\left(S_{1}\right) d S_{1}\right] d S_{2}
$$

Figure 1 exhibits visually the component reliability which is defined by the area where both tail curves interfere or overlap with each other. For the sake of clarity, (6a) and (6b) can be called the continuous stress-strength interference (CSSI) model.

If $S_{1}$ and $S_{2}$ are two discrete r.v. with the p.m.f. as follows,

$$
\begin{aligned}
& S_{1}=\left(S_{11}, S_{12}, \ldots, S_{1 k_{1}}\right), \\
& p_{1}=\left(p_{11}, p_{12}, \ldots, p_{1 k_{1}}\right), \\
& S_{2}=\left(S_{21}, S_{22}, \ldots, S_{2 k_{2}}\right), \\
& p_{2}=\left(p_{21}, p_{22}, \ldots, p_{2 k_{2}}\right),
\end{aligned}
$$

where $k_{1}$ and $k_{2}$ are, respectively, numbers of possible values that $S_{1}$ and $S_{2}$ can take on, then, according to Definition 1 , the UGF of $S_{1}$ and $S_{2}$ can be obtained as follows:

$$
\begin{aligned}
& u_{S_{1}}(z)=\sum_{j_{1}=1}^{k_{1}} p_{1 j_{1}} z^{S_{1 j_{1}}}, \\
& u_{S_{2}}(z)=\sum_{j_{2}=1}^{k_{2}} p_{2 j_{2}} z^{S_{2 j_{2}}} .
\end{aligned}
$$

If $f\left(S_{1}, S_{2}\right)$ is a function of $S_{1}$ and $S_{2}$, based on the UGF method introduced above, we can obtain the UGF of $f\left(S_{1}, S_{2}\right)$ as follows:

$$
\begin{aligned}
u_{f}(z) & =\bigotimes\left(u_{S_{1}}(z), u_{S_{2}}(z)\right) \\
& =\sum_{j_{1}=1}^{k_{1}} \sum_{j_{2}=1}^{k_{2}}\left(\prod_{i=1}^{2} p_{i j_{i}} z^{f\left(S_{1 j_{1}}, S_{2 j_{2}}\right)}\right)=\sum_{j=1}^{K} P_{j} z^{f_{j}},
\end{aligned}
$$

where $f_{j}$ and $P_{j}(j=1,2, \ldots, K)$ are possible values of function $f\left(S_{1}, S_{2}\right)$ and corresponding probabilities, respectively, and $K \leq k_{1} \times k_{2}$.
Definition 5 (discrete stress-strength interference (DSSI) model). If $f\left(S_{1}, S_{2}\right)=S_{2}-S_{1}$, the component reliability can be calculated as

$$
R=\operatorname{Pr}\left(f\left(S_{1}, S_{2}\right)>0\right)=\sum_{j=1}^{K} P_{j} \sigma\left(f_{j}\right) .
$$

Equation (10) is called the DSSI model, where $\sigma\left(f_{j}\right)$ is a binary-valued function with domain on the set of possible values of function $f\left(S_{1}, S_{2}\right)$ as

$$
\sigma\left(f_{j}\right)= \begin{cases}1, & f_{j}>0 \\ 0, & f_{j} \leq 0 .\end{cases}
$$

\section{Model Formulation}

In this section, a mathematical model is formulated for random forwarding actions for information diffusion over social networks. First, model description and notations used to develop the model are presented. Then, based on DSSI model introduced above, the effect (forwarding actions) of node is quantified as node susceptibility (NS). Finally, the most susceptible node is identified.

3.1. Model Description and Notations. In the model, one social network and $N$ nodes (users) of the social network are considered. User $n$ can receive different types of information (message) at different periods. Here, suppose that the unit of information is the piece. Let $M$ denote the number of types of information and $T$ the number of periods. Each period includes multiple time nodes, which divide the period into multiple equal time intervals. As shown in Figure 2, period $P_{t}$ includes $L_{t}$ time nodes $Q_{t j}, j=1,2, \ldots, L_{t}$, which divide the period into $L_{t}$ equal time intervals $\left[Q_{t, j-1}, Q_{t j}\right], j=$ $2,3, \ldots, L_{t}$ and $\left[Q_{t L_{t}}, Q_{t+1,1}\right]$. In time interval $\left[Q_{t, j-1}, Q_{t j}\right]$ at period $P_{t}$, for a piece of information, the user randomly forwards it or not. Whether to forward the information depends on several uncertain factors, including the interestingness of the information, the reliability of the information, egoistic motivation, and altruistic motivation. Whatever the motivation, forwarding actions indicate that the user is susceptible to the information, and the higher the frequency, the more susceptible the user. The decision problems addressed in this paper are as follows: (1) which users are most susceptible, (2) which types of information they are most susceptible to, and (3) when they are most susceptible.

For the sake of clarity of model description and development, we give the notations used to develop the model in Notation.

3.2. Quantifying Forwarding Actions Based on DSSI Model. In this subsection, definition of node (user) susceptibility (NS) is first given. Then, calculation steps of NS are presented.

3.2.1. Definition of Node Susceptibility (NS). As previously analyzed, forwarding actions indicate that the user is susceptible to the information, and the higher the frequency, 


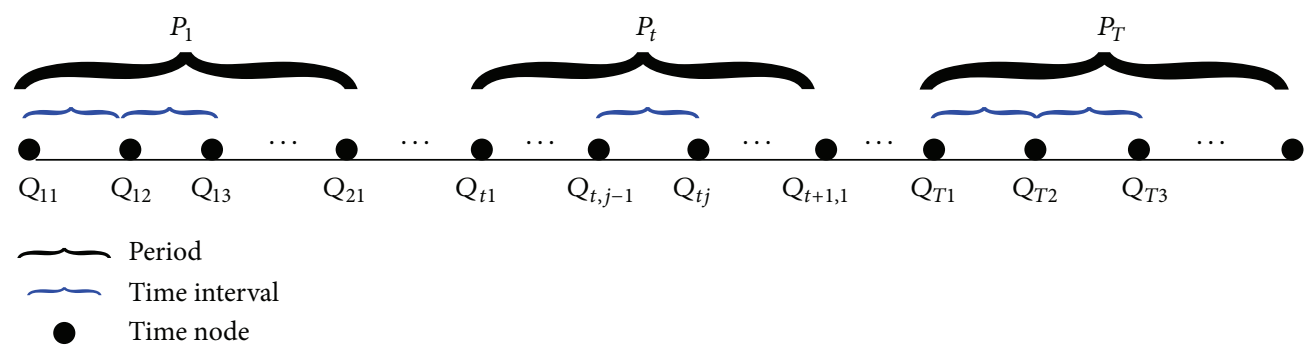

FIgURE 2: Modeling period and time node.
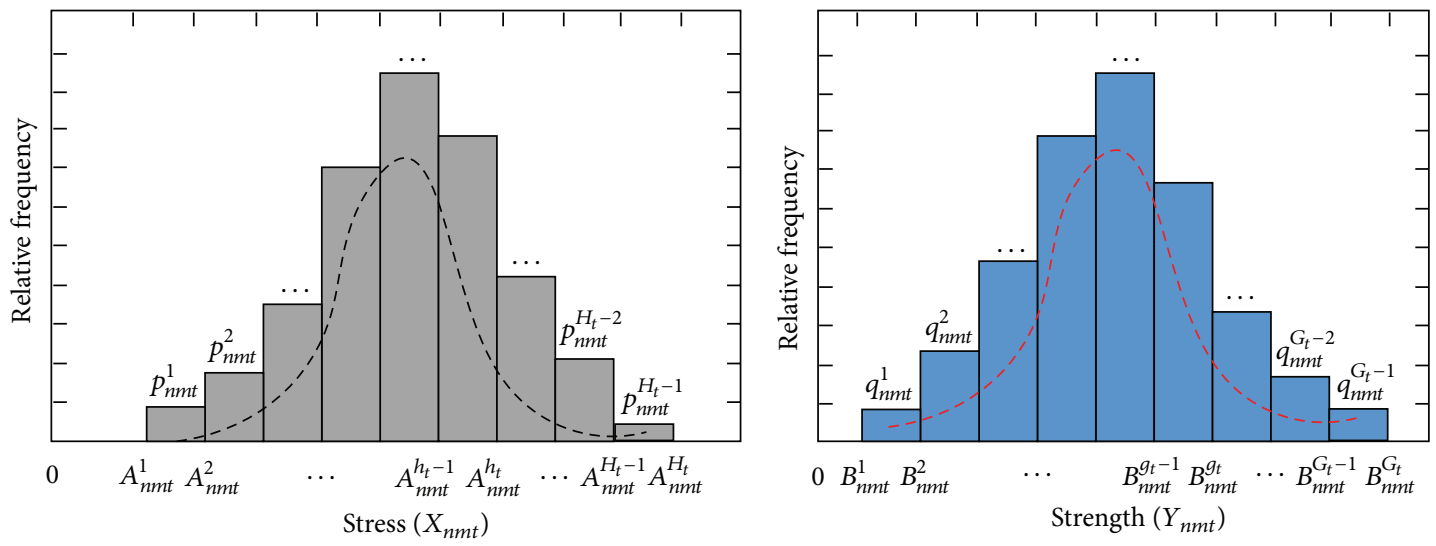

FIGURE 3: Histograms of stress and strength observations.

the more susceptible the user. To model the forwarding actions, a novel and universal criterion for the effect of user on information diffusion will be introduced, namely, node susceptibility (NS). NS is based on DSSI model and UGF method introduced in Section 2.

DSSI model considers two main random variables (r.v.): a stress which is any load applied on a component and a strength which is the maximum tolerance that the component can withstand without failing. To develop the model, this paper recognizes $U_{n}$ as equivalent to component, random quantity of $I_{m}$ that $U_{n}$ received at $P_{t}, X_{n m t}$ as equivalent to stress, and random quantity of $I_{m}$ that $U_{n}$ forwarded at $P_{t}$, $Y_{n m t}$, as equivalent to strength. For the sake of clarity, we give the following definition of NS.

Definition 6 (node susceptibility (NS)). Suppose that quantity of $I_{m}$ that $U_{n}$ received at $P_{t}, X_{n m t}$ and quantity of $I_{m}$ that $U_{n}$ forwarded at $P_{t}, Y_{n m t}$ are r.v. NS of $U_{n}$ in regard to $I_{m}$ at $P_{t}$ denoted by $\mathrm{NS}_{n m t}$ is the probability that $Y_{n m t}$ is larger than $X_{n m t}$. As a result, $\mathrm{NS}_{n m t}$ is given by

$$
\mathrm{NS}_{n m t}=\operatorname{Pr}\left(Y_{n m t}>X_{n m t}\right) \text {. }
$$

The following should be noted about the definition of NS:

(1) The statistic character of $X_{n m t}$ is based on a group of observations, that is, the observation parameters $A_{n m t i}$. Similarly, the statistic character of $Y_{n m t}$ is also based on a group of observations, that is, the observation parameters $B_{n m t j}$. This means that $X_{n m t}$ 's p.m.f. and $Y_{n m t}$ 's p.m.f. can be obtained from their observations, respectively.

(2) Since the observations are objective, $\mathrm{NS}_{n m t}$ is not dependent on the subjective judgments of the decision-makers. In addition, the more the observations are, the more accurate the evaluation is going to be.

(3) According to the definition of NS, random forwarding actions are quantified as a probability, which shows the degree of user's susceptibility to the corresponding information at the corresponding period. Therefore, based on NS, decision-maker can identify which users are most susceptible to the corresponding information at different periods.

3.2.2. Calculation Steps of NS. According to UGF method and DSSI model introduced previously, calculation steps of NS are given as follows.

Step 1 (deriving $X_{n m t}$ 's p.m.f. and $Y_{n m t}$ 's p.m.f.). Suppose that the observations of $X_{n m t}$ are $A_{n m t 1}, A_{n m t 2}, \ldots, A_{n m t L_{t}}$ and the observations of $Y_{n m t}$ are $B_{n m t 1}, B_{n m t 2}, \ldots, B_{n m t L_{t}}$. Two groups of observations can be described by histograms, as shown in Figure 3, and the class intervals of observations and their corresponding relative frequencies are obtained.

To obtain $X_{n m t}$ 's p.m.f., the midpoint values of each class interval $\left[A_{n m t}^{h_{t}-1}, A_{n m t}^{h_{t}}\right], h_{t}=2,3, \ldots, H_{t}$, are treated as possible values of $X_{n m t}$, and relative frequencies of each 
class interval $\left[A_{n m t}^{h_{t}-1}, A_{n m t}^{h_{t}}\right], h_{t}=2,3, \ldots, H_{t}$, are treated as corresponding probabilities. Thus, $X_{n m t}$ 's p.m.f. is obtained as follows:

$$
\begin{aligned}
& X_{n m t} \\
& =\left(\frac{A_{n m t}^{2}-A_{n m t}^{1}}{2}, \frac{A_{n m t}^{3}-A_{n m t}^{2}}{2}, \ldots, \frac{A_{n m t}^{H_{t}}-A_{n m t}^{H_{t}-1}}{2}\right) \\
& \triangleq \\
& =\left(X_{n m t}^{1}, X_{n m t}^{2}, \ldots, X_{n m t}^{H_{t}}\right), \\
& p_{n m t}=\left(p_{n m t}^{1}, p_{n m t}^{2}, \ldots, p_{n m t}^{H_{t}}\right),
\end{aligned}
$$

where $X_{n m t}^{h_{t}}=\left(A_{n m t}^{h_{t}}-A_{n m t}^{h_{t}-1}\right) / 2, h_{t}=1,2, \ldots, H_{t}$.

Similarly, the midpoint values of each class interval $\left[B_{n m t}^{g_{t}-1}, B_{n m t}^{g_{t}}\right], g_{t}=2,3, \ldots, G_{t}$, are treated as possible values of $Y_{n m t}$, and relative frequencies of each class interval $\left[B_{n m t}^{g_{t}-1}, B_{n m t}^{g_{t}}\right], g_{t}=2,3, \ldots, G_{t}$, are treated as corresponding probabilities. Thus, $Y_{n m t}$ 's p.m.f. is obtained as follows:

$$
\begin{aligned}
& Y_{n m t} \\
& \quad=\left(\frac{B_{n m t}^{2}-B_{n m t}^{1}}{2}, \frac{B_{n m t}^{3}-B_{n m t}^{2}}{2}, \ldots, \frac{B_{n m t}^{G_{t}}-B_{n m t}^{G_{t}-1}}{2}\right) \\
& \quad \triangleq\left(Y_{n m t}^{1}, Y_{n m t}^{2}, \ldots, Y_{n m t}^{G_{t}}\right), \\
& q_{n m t}=\left(q_{n m t}^{1}, q_{n m t}^{2}, \ldots, q_{n m t}^{G_{t}}\right),
\end{aligned}
$$

where $Y_{n m t}^{g_{t}}=\left(B_{n m t}^{g_{t}}-B_{n m t}^{g_{t}-1}\right) / 2, g_{t}=1,2, \ldots, G_{t}$.

Step 2 (deriving $X_{n m t}$ 's UGF, $Y_{n m t}$ 's UGF, and $f\left(X_{n m t}, Y_{n m t}\right.$ )'s UGF). According to Definition 1, the UGFs of $X_{n m t}$ and $Y_{n m t}$ can be given as follows:

$$
\begin{aligned}
u_{X_{n m t}}(z) & =p_{n m t}^{1} z^{X_{n m t}^{1}}+p_{n m t}^{2} z^{X_{n m t}^{2}}+\cdots+p_{n m t}^{H_{t}} z^{X_{n m t}^{H_{t}}} \\
& =\sum_{h_{t}=1}^{H_{t}} p_{n m t}^{h_{t}} z^{X_{n m t}^{h_{t}}}, \\
u_{Y_{n m t}}(z) & =q_{n m t}^{1} z^{Y_{n m t}^{1}}+q_{n m t}^{2} z^{Y_{n m t}^{2}}+\cdots+q_{n m t}^{G_{t}} z^{Y_{n m t}^{G_{t}}} \\
& =\sum_{g_{t}=1}^{G_{t}} q_{n m t}^{g_{t}} z^{Y_{n m t}^{g_{t}}} .
\end{aligned}
$$

Because $f\left(X_{n m t}, Y_{n m t}\right)$ is a function of $X_{n m t}$ and $Y_{n m t}$, based on Definitions 2 and 3, the UGF of $f\left(X_{n m t}, Y_{n m t}\right)$ can be obtained as follows:

$$
\begin{aligned}
u_{f}(z) & =\bigotimes\left(u_{X_{n m t}}(z), u_{Y_{n m t}}(z)\right) \\
& =\bigotimes\left(\sum_{h_{t}=1}^{H_{t}} p_{n m t}^{h_{t}} z^{X_{n m t}^{h_{t}}}, \sum_{g_{t}=1}^{G_{t}} q_{n m t}^{g_{t}} z^{Y_{n m t}^{g_{t}}}\right)
\end{aligned}
$$

$$
\begin{aligned}
& =\sum_{h_{t}=1}^{H_{t}} \sum_{g_{t}=1}^{G_{t}}\left(p_{n m t}^{h_{t}} z^{f\left(X_{n m t}^{h_{t}}, Y_{n m t}^{g_{t}}\right)} q_{n m t}^{g_{t}} z^{f\left(X_{n m t}^{h_{t}}, Y_{n m t}^{G_{t}}\right)}\right) \\
& =\sum_{r=1}^{R} \lambda_{r} z^{f_{r}}
\end{aligned}
$$

where $f_{r}$ and $\lambda_{r}(r=1,2, \ldots, R)$ are possible values of function $f\left(X_{n m t}, Y_{n m t}\right)$ and corresponding probabilities, respectively, and $R \leq H_{t} \times G_{t}$.

Step 3 (calculating $\mathrm{NS}_{n m t}$ based on DSSI model). Suppose that $f\left(X_{n m t}, Y_{n m t}\right)=Y_{n m t}-X_{n m t}$, according to Definition 5, $\mathrm{NS}_{n m t}$ can be calculated as

$$
\mathrm{NS}_{n m t}=\operatorname{Pr}\left(f\left(X_{n m t}, Y_{n m t}\right)>0\right)=\sum_{r=1}^{R} \lambda_{r} \sigma\left(f_{r}\right),
$$

where $\sigma\left(f_{r}\right)$ is a binary-valued function with domain on the set of possible values of function $\left(Y_{n m t}-X_{n m t}\right)$ as

$$
\sigma\left(f_{r}\right)= \begin{cases}1, & f_{r}>0 \\ 0, & f_{r} \leq 0\end{cases}
$$

3.3. Identifying the Most Susceptible User at Different Periods. Without loss of generality, we give the process of identifying the most susceptible user in regard to information $I_{m}$. Based on the above calculation of NS, we can obtain each user's NS in regard to $I_{m}$ at different periods, as shown in the following:

$$
\begin{gathered}
\mathrm{NS}_{1 m 1}, \mathrm{NS}_{2 m 1}, \ldots, \mathrm{NS}_{\mathrm{Nm1}}, \\
\mathrm{NS}_{1 m 2}, \mathrm{NS}_{2 m 2}, \ldots, \mathrm{NS}_{\mathrm{Nm} 2}, \\
\vdots \\
\mathrm{NS}_{1 m T}, \mathrm{NS}_{2 m T}, \ldots, \mathrm{NS}_{\mathrm{NmT}}, \\
\mathrm{NS}_{n_{1} m 1}=\max _{1 \leq n \leq N}\left\{\mathrm{NS}_{n m 1}\right\}, \quad 1 \leq n_{1} \leq N, \\
\mathrm{NS}_{n_{2} m 2}=\max _{1 \leq n \leq N}\left\{\mathrm{NS}_{n m 2}\right\}, \quad 1 \leq n_{2} \leq N, \\
\vdots \mathrm{NS}_{n_{T} m T}=\max _{1 \leq n \leq N}\left\{\mathrm{NS}_{n m T}\right\}, \quad 1 \leq n_{T} \leq N .
\end{gathered}
$$

Equations (19a1), (19a2), and (19aN) shows that at corresponding period, which user is most susceptible to information $I_{m}$. For example, at period $P_{1}$, user $U_{n_{1}}$ is most susceptible to information $I_{m}$, and at period $P_{T}$, user $U_{n_{T}}$ is most susceptible to information $I_{m}$. Therefore, based on each user's NS, three main questions are solved: (1) which user is most susceptible. (2) which type of information he is most susceptible to, and (3) when he is most susceptible. In real-life decision, more susceptible users can be extracted as needed. To this end, decision-makers (politicians 
or enterprises) only need to rank $\mathrm{NS}_{n m t}, t=1,2, \ldots, T$; and $n=1,2, \ldots, N$, and set thresholds to mine the top ranked ones and then extract the corresponding users. To achieve the most effective advocation/advertisement, at the corresponding period, politicians/enterprises can post the corresponding information to these users.

\section{Case Study}

This section aims to illustrate the feasibility and practicality of the proposed model through its application to a test case.

4.1. Case Description. The case study was motivated by the problem of extracting appropriate users for advertisement over a social network-Meituan. Meituan is a Chinese groupbuying website for locally found consumer products and retail services, and it sells vouchers from merchants for deals, subject to minimum number of buyers who demand a discount. Meituan generates most of its revenue from mobile application services, and it has partnering agreement with 400 thousand Chinese local businesses. In 2014, Meituan accounts for $60 \%$ of the market share of deal-of-the-day group-buying websites in China, and in 2015 it has 200 million users [36]. One of the goals of Meituan is to find the most appropriate consumers for merchants and to provide the most efficient Internet promotion [37]. To this end, the proposed model in this paper will be applied to address the problem. Based on the model, decision-maker can extract appropriate consumers for different advertisements (e.g., cate, entertainment, and shopping) at different periods and then develop the most efficient Internet promotion strategy.

Without loss of generality, in this case study, six candidate users (i.e., $N=6$ ) are under consideration, and three types of advertisement information (i.e., $M=3$ ) will be posted to them. The observation of random forwarding actions contains four periods (i.e., $T=4$ ), and each period contains thirty time nodes (i.e., $L_{t}=30, t=1,2,3,4$ ). The objective is to determine which two users should be extracted and which type of advertisement information should be posted to these users at the corresponding period. It should be noted that the dimension of candidate set of users in case study is much less than the actual number of the users. The setting of this parameter is mainly based on the following two considerations. On the one hand, the main purpose of conducting case study is demonstrating the application of the proposed model, and low-dimensional parameter setting helps to clearly demonstrate calculation process. On the other hand, in the proposed model, although the dimensions of parameter settings have effect on computational complexity, the effect is little, because there are many data processing tools for mass data under the environment of big data. In essence, the key step of calculation of NS is Step 1: deriving $X_{n m t}$ 's p.m.f. and $Y_{n m t}$ 's p.m.f., where the class intervals of observations and their corresponding relative frequencies can be obtained from the histograms of observations. In real life, when the amount of observations is massive, the histograms can be directly obtained by using Statistic Package for Social Science (SPSS, a widely used program for statistical analysis in social science) (see the Appendix). In this sense, the model implementation in practice is feasible.

For the sake of clarity, the observation parameters $A_{n m t j}$ and $B_{n m t j}$ are, respectively, listed in Tables 1 and 2.

4.2. Results and Analysis. Based on the calculation steps of NS introduced in Section 3, we can obtain each user's NS in regard to corresponding advertisement information at corresponding period. As an example of calculation steps, we give the calculation of $\mathrm{NS}_{123}$ as follows.

According to the observation parameters in Tables 1 and 2, we describe two groups of data (data in bold font) by histograms as shown in Figure 4. follows:

Therefore, $X_{123}$ 's p.m.f. and $Y_{123}$ 's p.m.f. are obtained as

$$
\begin{aligned}
& X_{123}=(22.5,27.5,32.5,37.5,42.5,47.5,52.5), \\
& p_{123}=(0.03,0.07,0.20,0.30,0.27,0.10,0.03), \\
& Y_{123}=(24.5,29.5,34.5,39.5,44.5,49.5,54.5), \\
& q_{123}=(0.07,0.10,0.17,0.30,0.20,0.13,0.03) .
\end{aligned}
$$

Thus, we have

$$
\begin{aligned}
\mathrm{NS}_{123} & =\operatorname{Pr}\left(f\left(X_{123}, Y_{123}\right)>0\right)=\sum_{r=1}^{R} \lambda_{r} \sigma\left(f_{r}\right) \\
= & 0.07 \times 0.03+0.10 \times(0.03+0.07)+0.17 \\
& \times(0.03+0.07+0.20)+0.30 \\
& \times(0.03+0.07+0.20+0.30)+0.20 \\
& \times(0.03+0.07+0.20+0.30+0.27)+0.13 \\
& \times(0.03+0.07+0.20+0.30+0.27+0.10)+0.03 \\
& \times(0.03+0.07+0.20+0.30+0.27+0.10+0.03) \\
= & 0.5778
\end{aligned}
$$

All NS $_{n m t}(n=1,2,3,4,5,6 ; m=1,2,3 ; t=1,2,3,4)$ can be obtained, as shown in Table 3, where the values in bold font are the maximum two values of each row. Figure 5 visually shows each user's NS in regard to corresponding advertisement information at different periods.

Table 3 and Figure 5 show which two users should be extracted and which type of advertisement information should be posted to them at the corresponding period. For example, at the first period, users $U_{3}$ and $U_{4}$ are most susceptible to information $I_{1}$, while users $U_{2}$ and $U_{6}$ are most susceptible to information $I_{2}$. At the second and third periods, users $U_{2}$ and $U_{4}$ are most susceptible to information $I_{1}$. Users $U_{3}$ and $U_{4}$ are most susceptible to information $I_{2}$ at the third and fourth periods. These indicate that on the one hand different users are susceptible to different types of information at the same period, and on the other hand the same user is susceptible to different types of information at different periods. On the whole, users $U_{2}, U_{3}, U_{4}$, and $U_{6}$ are more susceptible, and information $I_{1}$ and information $I_{2}$ are 
TABLE 1: Observation parameters $A_{n m t j}$.

\begin{tabular}{|c|c|c|c|c|c|c|c|}
\hline \multicolumn{4}{|c|}{$t=1$} & & \multicolumn{3}{|c|}{$t=2$} \\
\hline & $I_{1}$ & $I_{2}$ & $I_{3}$ & & $I_{1}$ & $I_{2}$ & $I_{3}$ \\
\hline$U_{1}$ & $\begin{array}{c}39,37,37,39 \\
43,49,44,39 \\
36,29,34,29 \\
33,39,37,29 \\
24,34,34,49 \\
53,43,37,39 \\
31,44,44,47 \\
43,42\end{array}$ & $\begin{array}{c}34,49,45,47 \\
38,40,35,39 \\
37,29,24,34 \\
38,38,37,40 \\
45,43,53,45 \\
38,29,34,34 \\
32,35,43,47 \\
44,45 \\
\end{array}$ & $\begin{array}{c}41,39,38,40 \\
41,39,36,44 \\
35,25,44,41 \\
26,35,26,31 \\
35,40,40,55 \\
51,50,40,30 \\
39,45,41,43 \\
45,51 \\
\end{array}$ & $U_{1}$ & $\begin{array}{c}37,36,38,37 \\
33,48,41,37 \\
39,27,33,28 \\
34,37,39,29 \\
23,33,33,47 \\
54,43,36,38 \\
33,41,43,47 \\
41,44\end{array}$ & $\begin{array}{c}54,49,33,44 \\
36,37,39,39 \\
39,36,42,41 \\
33,36,37,29 \\
23,34,53,38 \\
38,28,34,29 \\
31,43,42,47 \\
44,44\end{array}$ & $\begin{array}{c}44,47,35,48, \\
39,40,34,38, \\
39,27,25,33, \\
37,39,36,40, \\
43,44,51,37, \\
39,29,35,32, \\
33,34,44,47, \\
45,44\end{array}$ \\
\hline$U_{2}$ & $\begin{array}{c}39,36,39,29 \\
31,28,38,37 \\
44,39,35,49 \\
33,38,38,27 \\
22,32,34,46 \\
52,45,37,38 \\
34,47,43,49 \\
44,43 \\
\end{array}$ & $\begin{array}{c}33,49,54,44 \\
36,37,39,39 \\
39,36,42,41 \\
33,36,37,29 \\
23,34,53,38 \\
38,28,34,29 \\
31,43,42,47 \\
44,44 \\
\end{array}$ & $\begin{array}{c}34,48,44,43 \\
38,39,38,36 \\
36,37,42,44 \\
32,39,39,27 \\
23,33,54,39 \\
37,29,34,28 \\
33,43,41,49 \\
44,42\end{array}$ & $U_{2}$ & $\begin{array}{c}39,32,45,25 \\
29,35,38,38 \\
38,37,44,42 \\
33,49,45,48 \\
38,53,48,40 \\
37,25,35,35 \\
38,35,45,50 \\
30,43 \\
\end{array}$ & $\begin{array}{c}41,40,34,29, \\
27,34,49,40, \\
37,24,32,34, \\
34,49,39,50, \\
39,55,37,38, \\
38,42,44,45, \\
44,31,42,50, \\
45,40\end{array}$ & $\begin{array}{c}39,40,37,37, \\
43,45,50,41, \\
41,24,32,34, \\
32,44,43,50, \\
39,52,31,39, \\
41,27,27,35, \\
36,34,41,37, \\
40,40\end{array}$ \\
\hline$U_{3}$ & $\begin{array}{c}36,34,44,31, \\
32,28,39,41, \\
46,38,53,46, \\
31,37,35,27,21, \\
32,34,45,50 \\
38,36,39,43, \\
49,39,45,40, \\
41\end{array}$ & $\begin{array}{c}37,37,44,27 \\
35,29,38,39 \\
39,36,34,46 \\
31,37,37,28 \\
25,33,31,47 \\
51,44,37,39 \\
43,48,45,46 \\
44,44\end{array}$ & $\begin{array}{c}38,37,41,30 \\
32,27,39,37 \\
45,36,33,46 \\
34,37,36,29 \\
25,33,35,37 \\
52,43,37,39 \\
43,49,41,47 \\
38,41\end{array}$ & $U_{3}$ & $\begin{array}{c}37,39,42,24 \\
33,28,39,37 \\
43,38,34,49 \\
34,38,40,29 \\
24,33,33,48 \\
53,44,37,39 \\
44,47,42,49 \\
44,44\end{array}$ & $\begin{array}{c}38,37,44,28 \\
33,29,36,38 \\
42,38,32,48 \\
31,39,39,26 \\
24,34,31,49 \\
55,44,39,39 \\
31,46,41,47 \\
41,42\end{array}$ & $\begin{array}{c}36,36,37,27, \\
33,29,36,36, \\
41,37,34,48, \\
31,39,39,29, \\
24,33,34,46, \\
51,43,36,39, \\
31,41,42,47, \\
44,41\end{array}$ \\
\hline$U_{4}$ & $\begin{array}{c}37,40,38,37 \\
33,49,39,37 \\
43,30,34,28 \\
33,37,39,28 \\
24,34,35,47 \\
53,45,38,39 \\
44,50,51,47 \\
43,44\end{array}$ & $\begin{array}{c}38,39,43,26 \\
35,27,39,37 \\
37,37,33,46 \\
33,36,36,29 \\
25,33,32,46 \\
53,44,37,39 \\
44,49,41,47 \\
36,45\end{array}$ & $\begin{array}{c}37,39,37,28 \\
35,27,39,39 \\
45,38,33,49 \\
31,37,36,29 \\
24,33,32,47 \\
53,44,37,37 \\
44,49,42,52 \\
43,44\end{array}$ & $U_{4}$ & $\begin{array}{c}35,48,51,42 \\
39,38,38,39 \\
37,37,42,48 \\
31,37,37,27 \\
24,33,54,38 \\
36,26,34,28 \\
32,43,44,49 \\
44,41\end{array}$ & $\begin{array}{c}31,38,37,29 \\
24,34,33,49 \\
42,44,38,38 \\
39,38,37,38 \\
43,43,52,38 \\
36,29,34,30 \\
30,33,42,49 \\
45,41\end{array}$ & $\begin{array}{c}31,50,44,49 \\
36,39,31,37 \\
40,29,26,35 \\
37,39,37,39 \\
44,41,54,43 \\
37,24,34,35, \\
35,35,45,49 \\
41,41\end{array}$ \\
\hline$U_{5}$ & $\begin{array}{c}44,50,39,21 \\
31,35,35,47 \\
41,48,38,53 \\
35,39,41,30 \\
29,31,36,38 \\
37,40,45,44 \\
37,35,41,49 \\
45,45\end{array}$ & $\begin{array}{c}41,46,36,25 \\
35,31,35,44 \\
45,49,37,53 \\
35,40,41,29 \\
29,34,39,38 \\
40,40,43,44 \\
38,32,44,36 \\
45,41 \\
\end{array}$ & $\begin{array}{c}44,35,43,49 \\
40,55,39,40 \\
36,39,44,34 \\
39,43,42,23 \\
32,33,35,38 \\
41,29,27,35 \\
40,32,41,36 \\
41,46\end{array}$ & $U_{5}$ & $\begin{array}{c}39,37,38,37 \\
33,49,39,37 \\
43,30,34,28 \\
33,37,39,28 \\
24,34,35,47 \\
53,45,38,39 \\
44,50,51,47 \\
43,44\end{array}$ & $\begin{array}{c}38,38,43,26 \\
35,27,39,37 \\
37,37,33,46 \\
33,36,36,29 \\
25,33,32,46 \\
53,44,37,39 \\
44,49,41,47 \\
36,45\end{array}$ & $\begin{array}{c}38,37,39,28 \\
35,27,39,39 \\
45,38,33,49 \\
31,37,36,29 \\
24,33,32,47 \\
53,44,37,37 \\
44,49,42,52, \\
43,44\end{array}$ \\
\hline$U_{6}$ & $\begin{array}{c}38,44,38,27 \\
32,29,37,36 \\
41,39,31,49 \\
34,38,36,29 \\
24,34,31,49 \\
52,43,39,39 \\
41,53,41,49 \\
42,44 \\
\end{array}$ & $\begin{array}{c}39,39,36,29 \\
31,28,38,37 \\
44,39,35,49 \\
33,38,38,27 \\
22,32,34,46 \\
52,45,37,38 \\
34,47,43,49 \\
44,43\end{array}$ & $\begin{array}{c}39,37,37,29 \\
34,27,39,36 \\
41,39,31,46 \\
33,38,38,24 \\
24,31,32,46 \\
53,41,39,39 \\
33,41,44,49 \\
44,44\end{array}$ & $U_{6}$ & $\begin{array}{c}33,47,55,43 \\
36,40,37,39 \\
45,27,35,28 \\
38,37,39,37 \\
34,47,35,37 \\
39,30,24,34 \\
41,48,41,41 \\
38,42\end{array}$ & $\begin{array}{c}39,38,41,37 \\
33,50,36,38 \\
36,28,34,26 \\
32,37,37,26, \\
25,31,34,48 \\
52,43,38,36 \\
45,49,45,54 \\
45,34\end{array}$ & $\begin{array}{c}36,37,38,24, \\
45,27,34,42, \\
36,24,28,35, \\
37,38,45,36, \\
43,51,32,49, \\
46,44,39,39, \\
32,37,41,34, \\
42,31\end{array}$ \\
\hline \multicolumn{4}{|c|}{$t=3$} & & \multicolumn{3}{|c|}{$t=4$} \\
\hline & $I_{1}$ & $I_{2}$ & $I_{3}$ & & $I_{1}$ & $I_{2}$ & $I_{3}$ \\
\hline$U_{1}$ & $\begin{array}{c}36,51,53,43 \\
37,39,38,37 \\
36,39,43,49 \\
43,39,36,31 \\
34,29,35,39 \\
37,29,24,34 \\
30,44,43,47 \\
43,42\end{array}$ & $\begin{array}{c}33,47,42,47, \\
39,41,38,38, \\
37,40,45,43, \\
36,39,37,29, \\
24,34,51,45, \\
38,29,34,34, \\
33,35,43,47, \\
44,45\end{array}$ & $\begin{array}{c}43,38,38,43 \\
41,40,26,35 \\
25,31,35,40 \\
36,44,35,45 \\
44,41,39,55 \\
51,50,40,30 \\
39,44,40,40 \\
45,50\end{array}$ & $U_{1}$ & $\begin{array}{c}40,36,39,27 \\
33,28,35,36 \\
38,37,33,48 \\
34,37,38,29 \\
23,33,32,47 \\
54,44,36,38 \\
35,41,42,47 \\
41,43\end{array}$ & $\begin{array}{c}38,39,37,36 \\
42,41,32,36 \\
37,29,23,34 \\
55,49,35,44 \\
36,37,51,38 \\
38,28,34,29 \\
32,43,42,47 \\
44,41\end{array}$ & $\begin{array}{c}33,38,39,28, \\
25,33,46,47, \\
35,48,39,40, \\
38,39,37,40, \\
43,44,52,37, \\
39,29,35,32, \\
35,34,43,47 \\
45,36\end{array}$ \\
\hline
\end{tabular}


TABLE 1: Continued.

\begin{tabular}{|c|c|c|c|c|c|c|c|}
\hline$U_{2}$ & $\begin{array}{c}35,37,44,38 \\
35,49,39,36 \\
39,29,31,28 \\
32,38,38,27 \\
22,32,31,46 \\
52,45,37,38 \\
35,47,44,49 \\
44,43\end{array}$ & $\begin{array}{c}38,39,36,29 \\
31,28,36,37 \\
44,39,35,49 \\
33,38,38,27 \\
22,32,34,46 \\
52,45,37,38 \\
34,47,43,49 \\
44,43\end{array}$ & $\begin{array}{c}34,37,38,37 \\
33,49,39,37 \\
43,30,34,28 \\
33,37,39,28 \\
24,34,35,47 \\
53,45,38,39 \\
44,50,51,49 \\
43,44\end{array}$ & $U_{2}$ & $\begin{array}{c}37,38,39,37 \\
44,41,37,32 \\
45,26,29,35 \\
32,49,40,48 \\
38,53,47,44 \\
38,25,35,35 \\
39,35,45,50 \\
45,42\end{array}$ & $\begin{array}{c}50,40,38,24, \\
32,34,40,41, \\
34,29,27,34, \\
35,49,39,50 \\
38,55,38,38 \\
38,42,43,40, \\
45,31,42,50 \\
45,40\end{array}$ & $\begin{array}{c}51,40,41,24, \\
32,34,33,45, \\
43,50,39,52, \\
37,40,37,37, \\
43,45,32,39 \\
41,27,27,35, \\
38,34,41,37, \\
46,42\end{array}$ \\
\hline$U_{3}$ & $\begin{array}{c}38,32,45,25 \\
29,35,36,38 \\
38,37,44,42, \\
33,49,45,48, \\
38,53,48,44 \\
37,26,35,35 \\
38,35,45,50 \\
45,40\end{array}$ & $\begin{array}{c}42,35,43,49, \\
40,55,39,40, \\
36,39,44,34, \\
37,43,42,23, \\
32,33,35,38, \\
41,29,27,35, \\
46,32,41,36, \\
41,45\end{array}$ & $\begin{array}{c}55,49,33,44, \\
36,37,35,39, \\
39,36,42,41, \\
31,36,37,29 \\
23,34,52,38, \\
38,28,34,29 \\
30,43,42,47, \\
44,40\end{array}$ & $U_{3}$ & $\begin{array}{c}38,37,43,38 \\
34,48,36,39 \\
42,24,33,28 \\
35,38,40,29 \\
24,33,32,48 \\
53,44,37,39 \\
42,47,42,49 \\
44,45\end{array}$ & $\begin{array}{c}37,38,42,39 \\
32,48,32,39 \\
39,26,24,34, \\
37,36,44,28 \\
33,29,31,49 \\
55,44,39,39 \\
29,46,41,47 \\
41,40\end{array}$ & $\begin{array}{c}31,38,39,29, \\
24,33,37,36, \\
37,27,33,29, \\
35,36,41,37, \\
34,48,36,46, \\
51,43,36,39, \\
30,41,42,47, \\
45,41\end{array}$ \\
\hline$U_{4}$ & $\begin{array}{c}38,37,42,30 \\
34,29,39,40 \\
38,37,33,49 \\
35,37,39,28 \\
24,34,35,47 \\
53,45,38,39 \\
43,50,54,47 \\
43,44\end{array}$ & $\begin{array}{c}38,36,37,36 \\
33,46,32,36 \\
36,29,25,33 \\
38,37,43,26 \\
35,27,32,45 \\
53,44,37,39 \\
43,49,41,47 \\
36,45\end{array}$ & $\begin{array}{c}40,39,45,38 \\
33,49,36,39 \\
37,28,35,27 \\
30,37,36,29 \\
24,33,31,47 \\
53,44,37,37 \\
45,49,42,52 \\
43,44\end{array}$ & $U_{4}$ & $\begin{array}{c}36,37,38,27 \\
24,35,32,48 \\
51,42,39,39 \\
36,39,37,38 \\
42,48,53,38 \\
37,26,34,28 \\
33,43,44,49 \\
46,41\end{array}$ & $\begin{array}{c}38,39,37,38 \\
43,44,30,39 \\
37,29,24,34 \\
35,49,42,44 \\
38,39,51,38 \\
36,29,34,30 \\
35,33,44,49 \\
45,40\end{array}$ & $\begin{array}{c}30,54,44,49, \\
36,39,36,39, \\
37,39,44,41, \\
30,37,41,29, \\
23,35,52,43, \\
37,24,34,36, \\
36,35,45,48, \\
31,39\end{array}$ \\
\hline$U_{5}$ & $\begin{array}{c}36,47,41,48 \\
38,53,44,50, \\
39,21,31,35 \\
36,39,41,30 \\
29,31,36,38 \\
37,40,45,44, \\
38,35,41,49 \\
45,45\end{array}$ & $\begin{array}{c}36,44,45,49, \\
37,53,40,46, \\
36,25,35,31, \\
34,40,41,29, \\
29,34,37,38, \\
40,40,43,44, \\
39,32,44,36, \\
45,41\end{array}$ & $\begin{array}{c}37,40,36,39 \\
44,34,45,35, \\
43,49,40,55 \\
38,43,42,23 \\
32,33,35,38, \\
41,29,27,35, \\
41,32,41,36 \\
40,40\end{array}$ & $U_{5}$ & $\begin{array}{c}38,37,36,37 \\
33,48,32,37 \\
39,28,24,34 \\
38,37,43,30 \\
34,28,36,47 \\
53,45,39,39 \\
45,50,51,47 \\
43,45\end{array}$ & $\begin{array}{c}38,37,36,37 \\
33,46,35,36 \\
36,29,25,33 \\
37,38,43,26 \\
35,27,35,46 \\
53,44,37,39 \\
44,48,41,47 \\
36,44\end{array}$ & $\begin{array}{c}38,39,45,38 \\
33,48,29,37 \\
36,29,24,33 \\
39,37,39,29 \\
35,27,35,47 \\
53,44,37,37 \\
47,49,42,52 \\
43,39\end{array}$ \\
\hline$U_{6}$ & $\begin{array}{c}36,37,41,39 \\
31,49,39,44 \\
38,27,32,29 \\
35,38,36,29 \\
24,34,30,49 \\
52,43,39,39 \\
40,53,41,49 \\
42,44\end{array}$ & $\begin{array}{c}39,37,44,39 \\
35,49,37,38 \\
36,29,31,29 \\
34,38,38,27 \\
22,32,33,46 \\
52,45,37,38 \\
32,47,44,49 \\
44,43\end{array}$ & $\begin{array}{c}40,36,41,37 \\
31,46,34,38 \\
39,24,24,31 \\
38,37,37,29 \\
34,27,33,46 \\
53,41,39,39 \\
32,41,44,49 \\
44,45\end{array}$ & $U_{6}$ & $\begin{array}{c}38,39,45,25 \\
35,28,35,37 \\
39,37,34,47 \\
34,47,55,43, \\
36,40,36,37, \\
39,30,27,34 \\
44,48,41,41 \\
38,40\end{array}$ & $\begin{array}{c}32,38,37,26 \\
25,31,38,39 \\
41,37,33,50 \\
37,38,36,29 \\
34,26,35,48 \\
52,43,38,37 \\
47,45,45,54 \\
45,35\end{array}$ & $\begin{array}{c}35,42,36,24, \\
28,35,37,38, \\
44,36,43,51, \\
37,37,38,24 \\
45,27,34,49 \\
46,44,39,39 \\
31,37,41,34 \\
42,38\end{array}$ \\
\hline
\end{tabular}
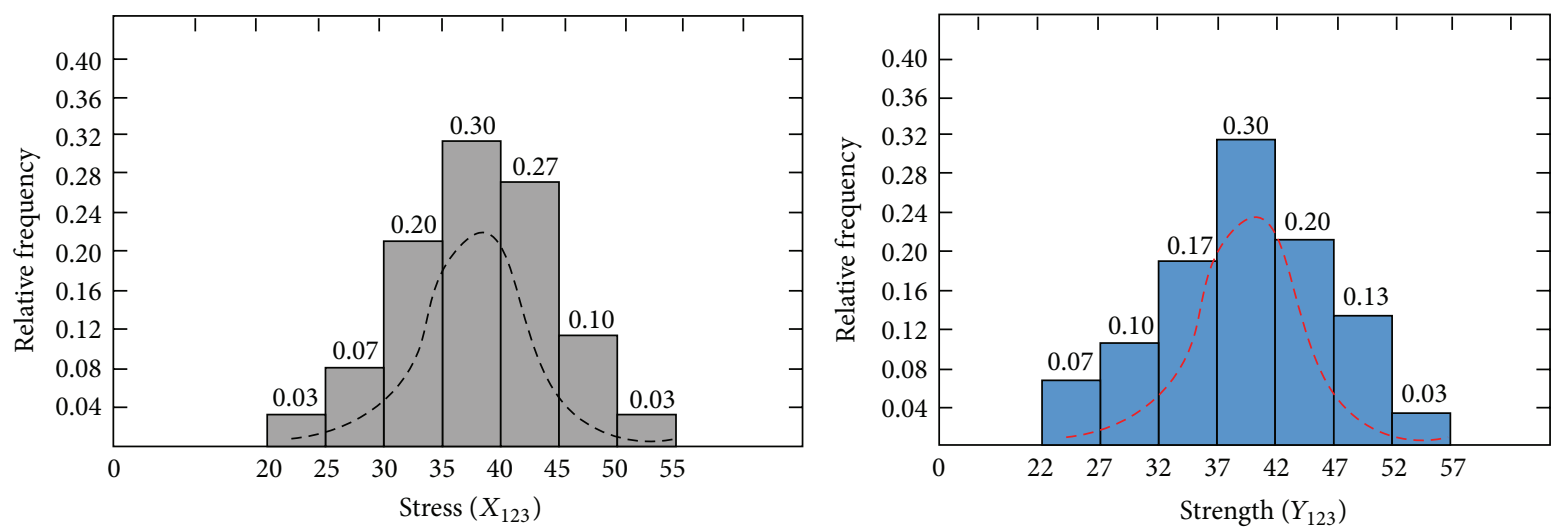

Figure 4: Histograms of $X_{123}$ and $Y_{123}$. 
TABLE 2: Observation parameters $B_{n m t j}$.

\begin{tabular}{|c|c|c|c|c|c|c|c|}
\hline \multicolumn{4}{|c|}{$t=1$} & & \multicolumn{3}{|c|}{$t=2$} \\
\hline & $I_{1}$ & $I_{2}$ & $I_{3}$ & & $I_{1}$ & $I_{2}$ & $I_{3}$ \\
\hline$U_{1}$ & $\begin{array}{c}39,53,50,51 \\
39,29,36,45 \\
34,41,34,39 \\
27,36,25,30 \\
30,39,38,40 \\
41,39,49,50 \\
42,45,39,44 \\
45,53\end{array}$ & $\begin{array}{c}37,46,35,39 \\
34,38,27,36 \\
25,31,36,40 \\
42,53,51,50 \\
39,33,44,39 \\
41,41,50,51 \\
45,45,42,45 \\
46,52\end{array}$ & $\begin{array}{c}42,40,41,39 \\
48,49,30,36 \\
25,30,37,40 \\
37,53,48,52 \\
40,32,34,45 \\
35,46,35,41 \\
42,45,39,46 \\
44,54\end{array}$ & $U_{1}$ & $\begin{array}{c}34,45,51,44 \\
37,38,36,35 \\
40,28,35,24 \\
38,44,43,35 \\
34,57,33,34 \\
37,29,42,30 \\
43,49,43,46 \\
40,41\end{array}$ & $\begin{array}{c}37,39,39,41 \\
46,51,23,36, \\
25,29,31,40, \\
40,56,54,48, \\
40,30,34,47 \\
36,39,34,41, \\
44,43,39,46, \\
44,52\end{array}$ & $\begin{array}{c}40,41,40,39 \\
51,49,25,35, \\
27,31,34,41, \\
42,56,51,50, \\
41,31,37,46, \\
36,44,36,38, \\
43,46,39,45, \\
46,52\end{array}$ \\
\hline$U_{2}$ & $\begin{array}{c}31,33,26,31 \\
35,38,37,45 \\
35,45,35,42 \\
41,55,49,50 \\
40,29,41,43 \\
40,40,46,45 \\
39,45,38,43 \\
46,51 \\
\end{array}$ & $\begin{array}{c}45,38,38,39 \\
41,45,38,54, \\
51,50,42,31 \\
36,46,35,46 \\
44,41,27,36 \\
26,29,35,38 \\
42,45,40,43 \\
46,51 \\
\end{array}$ & $\begin{array}{c}42,57,52,50 \\
44,35,35,47 \\
35,47,46,41 \\
48,40,39,44 \\
37,44,27,36 \\
26,31,37,40 \\
43,46,38,44 \\
45,47\end{array}$ & $U_{2}$ & $\begin{array}{c}41,40,41,45 \\
39,45,24,36 \\
26,31,30,39 \\
42,51,55,48 \\
36,39,33,42 \\
32,33,37,38 \\
43,47,41,48 \\
46,55\end{array}$ & $\begin{array}{c}40,38,39,41, \\
47,46,35,46, \\
36,44,44,40, \\
31,36,25,30, \\
36,41,41,54, \\
50,50,41,31, \\
37,46,41,45, \\
46,50\end{array}$ & $\begin{array}{c}32,43,43,50, \\
39,52,39,41 \\
37,37,43,45, \\
51,41,41,24, \\
32,34,32,39 \\
41,55,27,35, \\
37,34,41,37 \\
43,42\end{array}$ \\
\hline$U_{3}$ & $\begin{array}{c}37,45,34,40 \\
35,40,39,55, \\
51,48,37,32, \\
25,37,41,29, \\
31,40,41,39 \\
39,41,49,41 \\
45,43,41,44, \\
46,53\end{array}$ & $\begin{array}{c}26,35,41,32 \\
29,38,41,39 \\
39,40,48,39 \\
39,54,52,49 \\
38,31,33,45 \\
35,39,36,41 \\
43,44,26,45 \\
45,43\end{array}$ & $\begin{array}{c}39,54,51,49 \\
38,31,41,38 \\
39,41,48,38 \\
34,45,36,39 \\
37,38,35,36 \\
42,28,31,38 \\
44,45,27,44 \\
45,47\end{array}$ & $U_{3}$ & $\begin{array}{c}32,44,35,46, \\
34,41,28,36, \\
25,30,36,40, \\
37,53,48,51, \\
40,30,42,40, \\
41,39,48,48, \\
42,45,38,46, \\
44,53\end{array}$ & $\begin{array}{c}40,39,38,40, \\
41,45,35,44, \\
35,45,44,41, \\
27,35,26,31, \\
35,40,41,55, \\
51,50,40,30, \\
38,45,41,43, \\
45,50\end{array}$ & $\begin{array}{c}57,36,38,27 \\
33,28,35,39 \\
41,37,34,48, \\
30,39,39,29 \\
24,33,35,46, \\
51,43,36,38, \\
30,41,42,47 \\
46,41\end{array}$ \\
\hline$U_{4}$ & $\begin{array}{c}42,44,42,45, \\
54,47,38,57, \\
49,51,41,40, \\
35,46,35,45, \\
46,47,29,36, \\
30,31,41,35, \\
48,41,38,47, \\
37,27\end{array}$ & $\begin{array}{c}47,54,52,48, \\
46,39,37,46, \\
42,42,25,45, \\
53,42,39,45, \\
35,46,36,46, \\
34,47,38,42, \\
28,35,32,31 \\
41,37\end{array}$ & $\begin{array}{c}48,41,38,46, \\
41,43,36,45, \\
36,47,46,27, \\
42,46,39,44, \\
54,42,50,46, \\
52,49,45,39 \\
31,34,32,32 \\
40,37\end{array}$ & $U_{4}$ & $\begin{array}{c}40,38,41,39 \\
49,48,33,45 \\
35,46,36,41 \\
30,36,25,32 \\
36,40,41,55 \\
49,51,40,31 \\
40,45,38,46 \\
44,54\end{array}$ & $\begin{array}{c}47,38,39,39, \\
40,45,38,54, \\
51,50,41,32, \\
34,46,35,46, \\
44,40,27,36, \\
26,29,35,38, \\
42,45,40,43, \\
46,51\end{array}$ & $\begin{array}{c}30,50,44,49, \\
37,39,38,39, \\
37,39,44,41 \\
33,37,40,29 \\
23,35,53,43, \\
37,24,34,35, \\
36,35,45,49, \\
41,39\end{array}$ \\
\hline$U_{5}$ & $\begin{array}{c}42,39,40,40 \\
48,52,39,56, \\
52,49,39,31 \\
26,36,42,31 \\
30,41,37,46 \\
34,38,36,41 \\
45,45,39,45, \\
47,53\end{array}$ & $\begin{array}{c}32,48,36,39 \\
36,38,38,40 \\
38,42,45,41 \\
37,53,51,47 \\
38,33,27,37 \\
41,32,31,41 \\
42,43,41,48 \\
46,53\end{array}$ & $\begin{array}{c}38,53,50,48, \\
39,31,36,45 \\
35,40,37,38 \\
40,39,38,40 \\
49,41,36,35 \\
40,29,31,39 \\
45,43,26,46 \\
56,46\end{array}$ & $U_{5}$ & $\begin{array}{c}38,39,43,30 \\
34,28,34,37 \\
39,28,24,34 \\
39,39,38,37 \\
33,49,34,47 \\
53,45,38,39 \\
43,50,51,47 \\
43,45\end{array}$ & $\begin{array}{c}37,39,37,39 \\
33,46,33,36 \\
36,29,25,33 \\
39,38,43,26 \\
35,29,31,46 \\
53,44,38,39 \\
45,49,41,47 \\
39,45\end{array}$ & $\begin{array}{c}39,37,39,28 \\
35,27,32,37, \\
36,29,24,33 \\
31,47,53,44 \\
38,38,37,39 \\
45,38,33,49 \\
42,49,42,52 \\
43,44\end{array}$ \\
\hline$U_{6}$ & $\begin{array}{c}39,54,51,49, \\
38,28,23,35, \\
27,31,29,41 \\
33,44,34,38 \\
37,38,38,41 \\
40,41,48,50 \\
42,46,41,45 \\
46,53\end{array}$ & $\begin{array}{c}41,38,42,43 \\
49,52,24,36 \\
27,31,28,44 \\
36,55,50,48 \\
38,29,32,46 \\
36,40,37,39 \\
43,45,42,44 \\
48,53\end{array}$ & $\begin{array}{c}39,42,39,42 \\
47,51,25,35 \\
27,33,30,39 \\
42,53,50,48 \\
39,29,33,45 \\
32,39,36,37 \\
43,45,39,44 \\
47,54\end{array}$ & $U_{6}$ & $\begin{array}{c}40,37,39,37, \\
34,47,34,47, \\
56,43,36,40, \\
38,39,45,27, \\
35,28,36,37, \\
39,30,24,34, \\
40,48,41,41, \\
38,42\end{array}$ & $\begin{array}{c}37,38,36,28 \\
34,26,31,39 \\
39,26,25,31 \\
40,38,41,39 \\
33,50,35,48 \\
52,43,38,36 \\
44,39,45,54 \\
45,35\end{array}$ & $\begin{array}{c}38,38,45,36, \\
43,49,39,37, \\
38,24,45,57 \\
34,42,36,24, \\
28,35,31,49 \\
46,44,39,39 \\
33,39,41,34 \\
42,31\end{array}$ \\
\hline & & $t=3$ & & & & $t=4$ & \\
\hline & $I_{1}$ & $I_{2}$ & $I_{3}$ & & $I_{1}$ & $I_{2}$ & $I_{3}$ \\
\hline$U_{1}$ & $\begin{array}{c}32,37,40,29 \\
23,35,38,39 \\
38,39,44,41 \\
33,50,44,49 \\
36,39,53,43 \\
38,24,34,35 \\
36,35,45,49 \\
41,42\end{array}$ & $\begin{array}{c}39,45,42,45, \\
55,27,48,25, \\
51,49,32,41, \\
37,46,34,44, \\
38,45,49,42, \\
39,45,39,39, \\
33,34,31,30, \\
41,35\end{array}$ & $\begin{array}{c}38,47,34,46 \\
46,46,41,56 \\
50,51,42,38 \\
42,45,42,42, \\
45,44,28,35 \\
28,32,41,36 \\
51,42,39,45 \\
37,26\end{array}$ & $U_{1}$ & $\begin{array}{c}37,46,35,46, \\
40,46,39,44, \\
42,41,52,43, \\
42,53,52,48, \\
44,38,27,34, \\
32,30,40,35, \\
53,41,38,41 \\
35,47\end{array}$ & $\begin{array}{c}26,36,28,30 \\
42,37,43,47, \\
39,42,44,45, \\
42,56,51,51 \\
43,36,33,46 \\
35,41,47,42, \\
51,41,38,42, \\
36,42\end{array}$ & $\begin{array}{c}41,39,39,41, \\
49,41,39,55, \\
51,48,38,32, \\
27,37,41,30, \\
31,40,36,45, \\
34,39,35,40, \\
45,43,41,44, \\
46,53\end{array}$ \\
\hline
\end{tabular}


TABLe 2: Continued.

\begin{tabular}{|c|c|c|c|c|c|c|c|}
\hline$U_{2}$ & $\begin{array}{c}33,47,53,44 \\
38,39,39,37 \\
39,28,35,27 \\
39,39,45,38 \\
33,49,32,38 \\
36,29,24,33 \\
43,49,42,52 \\
43,45\end{array}$ & $\begin{array}{c}48,41,38,46, \\
41,44,34,45, \\
36,47,46,42, \\
42,46,39,44, \\
54,42,53,46, \\
52,49,45,39 \\
27,34,32,32, \\
40,37\end{array}$ & $\begin{array}{c}40,44,42,45, \\
54,47,38,57, \\
49,51,41,40, \\
35,42,35,45, \\
46,47,29,36, \\
30,31,41,35 \\
48,41,38,47, \\
37,26\end{array}$ & $U_{2}$ & $\begin{array}{c}36,46,34,47, \\
44,42,42,54, \\
52,49,46,39 \\
38,46,42,47 \\
52,40,53,42, \\
39,41,35,46 \\
27,35,32,31 \\
41,36\end{array}$ & $\begin{array}{c}27,35,26,31, \\
35,40,40,39 \\
38,40,41,45, \\
35,44,35,45, \\
44,41,40,54, \\
51,50,40,30 \\
38,45,41,43, \\
45,51\end{array}$ & $\begin{array}{c}38,55,50,49, \\
39,31,42,38, \\
39,40,48,42, \\
26,36,40,31, \\
30,41,36,46, \\
35,38,36,41, \\
44,45,40,45, \\
47,55\end{array}$ \\
\hline$U_{3}$ & $\begin{array}{c}33,49,46,44 \\
39,38,35,38, \\
38,28,45,27 \\
34,53,36,24 \\
28,35,39,38 \\
45,38,43,49 \\
34,38,41,34 \\
42,33\end{array}$ & $\begin{array}{c}47,43,51,48, \\
44,40,38,48, \\
35,46,45,44, \\
37,44,40,45, \\
56,41,49,42, \\
39,42,40,45, \\
33,37,30,29, \\
41,35\end{array}$ & $\begin{array}{c}37,43,41,46, \\
54,45,44,56, \\
50,49,42,39, \\
36,45,35,47, \\
42,42,28,35, \\
31,29,40,34, \\
51,42,39,44, \\
34,26\end{array}$ & $U_{3}$ & $\begin{array}{c}48,45,49,51 \\
45,42,39,42 \\
37,43,43,45 \\
48,39,39,36 \\
38,45,42,45 \\
42,46,55,38 \\
25,36,30,29 \\
39,35\end{array}$ & $\begin{array}{c}36,46,35,46, \\
44,40,38,54, \\
51,50,41,31 \\
47,38,38,39 \\
40,45,27,36 \\
26,29,35,38 \\
42,45,40,43, \\
46,51\end{array}$ & $\begin{array}{c}34,47,36,39, \\
35,39,38,40, \\
38,41,49,40, \\
39,53,51,48, \\
38,32,25,36, \\
41,32,31,42, \\
42,43,41,47, \\
46,54\end{array}$ \\
\hline$U_{4}$ & $\begin{array}{c}41,39,41,42, \\
48,51,27,35, \\
41,32,29,40, \\
39,55,51,48, \\
38,29,35,45 \\
33,39,37,39 \\
45,46,40,46 \\
45,54\end{array}$ & $\begin{array}{c}40,39,38,41, \\
49,40,37,55, \\
51,48,38,32, \\
27,37,41,30 \\
31,40,35,45, \\
34,39,35,40 \\
43,43,41,45 \\
46,53\end{array}$ & $\begin{array}{c}36,45,35,39 \\
36,41,41,38 \\
39,40,48,39 \\
38,54,52,49 \\
38,31,26,35 \\
41,30,29,39 \\
46,44,26,45 \\
45,43\end{array}$ & $U_{4}$ & $\begin{array}{c}41,39,41,42, \\
48,51,26,35, \\
41,32,29,40, \\
38,55,51,47, \\
38,29,33,45 \\
33,39,37,39 \\
44,46,40,46 \\
45,55\end{array}$ & $\begin{array}{c}41,39,39,41 \\
49,41,37,55 \\
51,48,38,32 \\
26,37,41,30 \\
31,40,37,45 \\
34,39,35,40 \\
43,43,41,44 \\
46,53\end{array}$ & $\begin{array}{c}33,45,35,39, \\
36,41,42,39, \\
38,40,48,39, \\
39,53,52,37, \\
38,31,26,35, \\
41,30,29,39, \\
43,44,26,45, \\
45,43\end{array}$ \\
\hline$U_{5}$ & $\begin{array}{c}35,46,35,38 \\
36,41,42,38, \\
39,40,48,42, \\
38,55,50,49, \\
39,31,27,36, \\
40,31,30,41 \\
44,44,40,45, \\
47,55\end{array}$ & $\begin{array}{c}39,53,51,49 \\
39,32,35,46 \\
34,38,37,40 \\
41,38,40,41 \\
48,38,27,36 \\
41,31,29,38 \\
45,44,25,46 \\
46,43\end{array}$ & $\begin{array}{c}36,37,40,29 \\
25,42,39,40 \\
38,40,49,39 \\
34,46,37,38 \\
37,54,38,53 \\
50,49,39,32 \\
42,43,26,43 \\
46,45\end{array}$ & $U_{5}$ & $\begin{array}{c}52,42,39,45 \\
35,46,45,54, \\
52,49,46,39 \\
38,46,42,42, \\
55,45,34,41 \\
34,40,42,47 \\
27,35,32,31 \\
41,37\end{array}$ & $\begin{array}{c}48,41,38,46, \\
41,42,34,45, \\
36,47,46,27, \\
42,46,39,44, \\
54,42,52,46, \\
52,49,45,39, \\
31,34,32,32, \\
40,36\end{array}$ & $\begin{array}{c}42,45,42,36, \\
55,38,42,39, \\
38,46,38,45, \\
39,34,37,43, \\
43,45,48,45, \\
49,51,45,42, \\
26,36,30,29, \\
39,33\end{array}$ \\
\hline$U_{6}$ & $\begin{array}{c}32,34,38,29 \\
23,30,39,35 \\
40,28,35,25 \\
38,44,43,35 \\
35,47,35,45 \\
50,48,38,39 \\
41,49,43,56 \\
40,42\end{array}$ & $\begin{array}{c}37,39,39,40, \\
46,51,25,36, \\
25,29,31,40, \\
42,56,54,48, \\
40,30,36,47, \\
36,39,34,41, \\
45,42,39,46, \\
44,52\end{array}$ & $\begin{array}{c}42,41,40,41, \\
51,48,25,34, \\
26,31,34,41, \\
42,56,51,50, \\
41,31,37,46, \\
36,45,36,38, \\
43,46,38,45, \\
46,53\end{array}$ & $U_{6}$ & $\begin{array}{c}39,43,41,42, \\
54,45,44,56, \\
50,49,42,39, \\
34,45,35,47, \\
45,42,29,35 \\
31,29,40,34, \\
51,42,39,44, \\
34,27\end{array}$ & $\begin{array}{c}37,44,41,27, \\
56,46,50,41, \\
38,46,38,42, \\
48,41,50,48, \\
43,41,37,45, \\
33,40,43,44, \\
32,36,32,32, \\
42,34\end{array}$ & $\begin{array}{c}38,44,40,45, \\
56,41,49,42, \\
39,47,40,45, \\
37,47,35,46, \\
45,41,48,42, \\
51,48,44,40, \\
32,37,30,27, \\
41,34\end{array}$ \\
\hline
\end{tabular}

TABLE 3: $\mathrm{NS}_{n m t}(n=1,2,3,4,5,6 ; m=1,2,3 ; t=1,2,3,4)$.

\begin{tabular}{|c|c|c|c|c|c|c|c|c|c|c|c|c|c|}
\hline \multicolumn{7}{|c|}{$t=1$} & \multicolumn{7}{|c|}{$t=2$} \\
\hline NS & $U_{1}$ & $U_{2}$ & $U_{3}$ & $U_{4}$ & $U_{5}$ & $U_{6}$ & NS & $U_{1}$ & $U_{2}$ & $U_{3}$ & $U_{4}$ & $U_{5}$ & $U_{6}$ \\
\hline$I_{1}$ & 0.5989 & 0.5344 & 0.6211 & 0.6178 & 0.5933 & 0.5778 & $I_{1}$ & 0.5911 & 0.5922 & 0.5844 & 0.6267 & 0.5667 & 0.5267 \\
\hline$I_{2}$ & 0.5667 & 0.6200 & 0.5844 & 0.6089 & 0.5800 & 0.6289 & $I_{2}$ & 0.5889 & 0.5900 & 0.6122 & 0.6656 & 0.5422 & 0.5322 \\
\hline$I_{3}$ & 0.5789 & 0.6533 & 0.5111 & 0.6311 & 0.6122 & 0.6100 & $I_{3}$ & 0.6322 & 0.5589 & 0.5222 & 0.5444 & 0.5244 & 0.5689 \\
\hline \multicolumn{7}{|c|}{$t=3$} & \multicolumn{7}{|c|}{$t=4$} \\
\hline NS & $U_{1}$ & $U_{2}$ & $U_{3}$ & $U_{4}$ & $U_{5}$ & $U_{6}$ & NS & $U_{1}$ & $U_{2}$ & $U_{3}$ & $U_{4}$ & $U_{5}$ & $U_{6}$ \\
\hline$I_{1}$ & 0.5356 & 0.5856 & 0.5067 & 0.6111 & 0.5711 & 0.5400 & $I_{1}$ & 0.6700 & 0.6156 & 0.6356 & 0.6022 & 0.6122 & 0.6400 \\
\hline$I_{2}$ & 0.5778 & 0.6511 & 0.6456 & 0.6167 & 0.5900 & 0.5956 & $I_{2}$ & 0.5822 & 0.6056 & 0.6322 & 0.6433 & 0.6211 & 0.6222 \\
\hline$I_{3}$ & 0.5889 & 0.6067 & 0.6244 & 0.5844 & 0.6278 & 0.6378 & $I_{3}$ & 0.6378 & 0.5956 & 0.6300 & 0.5844 & 0.6200 & 0.6756 \\
\hline
\end{tabular}



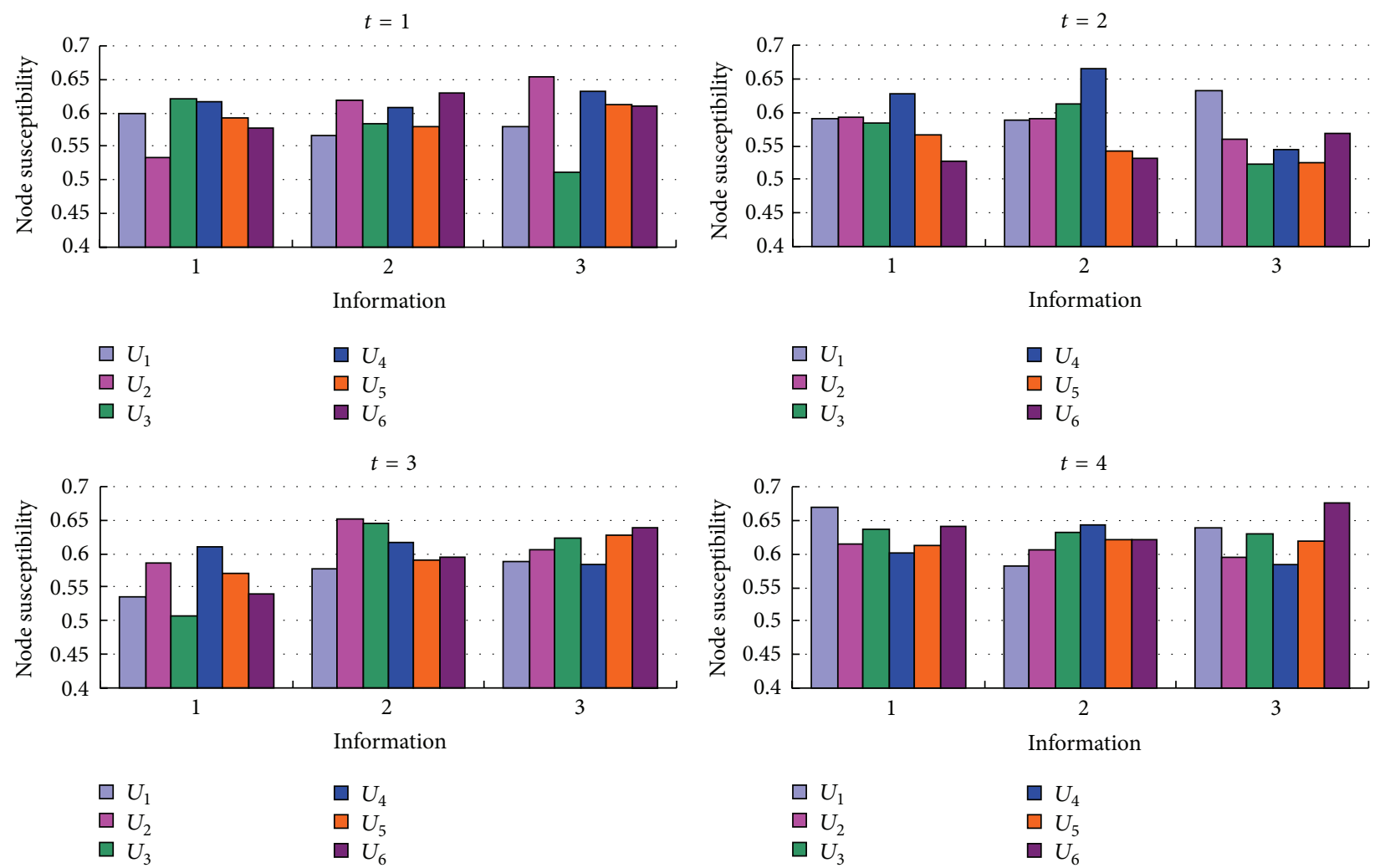

FIGURE 5: User's NS in regard to the corresponding information at different periods.

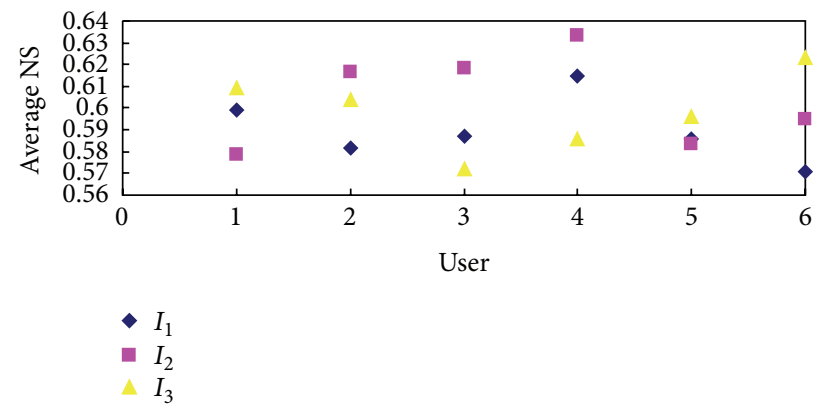

FIGURE 6: User's average NS.

more attractive. To quantitatively perform further analysis on the conclusion mentioned above, we calculate user's average NS and information's average NS, respectively, shown in Figures 6 and 7.

It can be seen from Figure 6 that, in regard to information $I_{1}, U_{4}$ is the most susceptible user, followed by $U_{1}$. In regard to information $I_{2}, U_{4}$ is also the most susceptible user, followed by $U_{3}$. In regard to information $I_{3}, U_{6}$ is the most susceptible user, followed by $U_{1}$. Compared with other users, $U_{5}$ is the least susceptible user, and he seems to be susceptible to none of these types of information. Indeed, information $I_{2}$ and information $I_{1}$ are more attractive, which can also be shown in Figure 7. Moreover, Figure 7 shows that values of average NS of three types of information at the fourth period are the

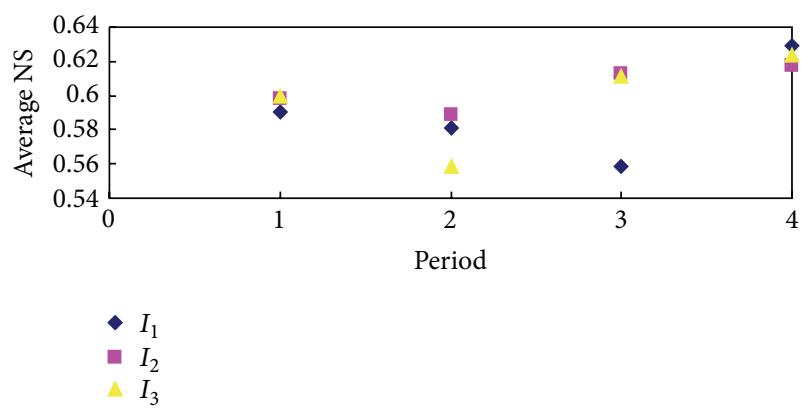

FIGURE 7: Information's average NS.

highest, which means that advertisement at this period will be most effective.

4.3. Comparison with Other Methods of Measuring User Susceptibility in the Existing Literature. To comprehensively analyze the proposed method for measuring user susceptibility, in this subsection, we will give the comparison with other methods in the existing literature [33-35]. The comparison includes quantified items, quantitative measures, mathematical model, key input parameters for decisions, implementation process in practice, and decision objectives. For the sake of clarity, the results of the comparison are displayed in Table 4. 


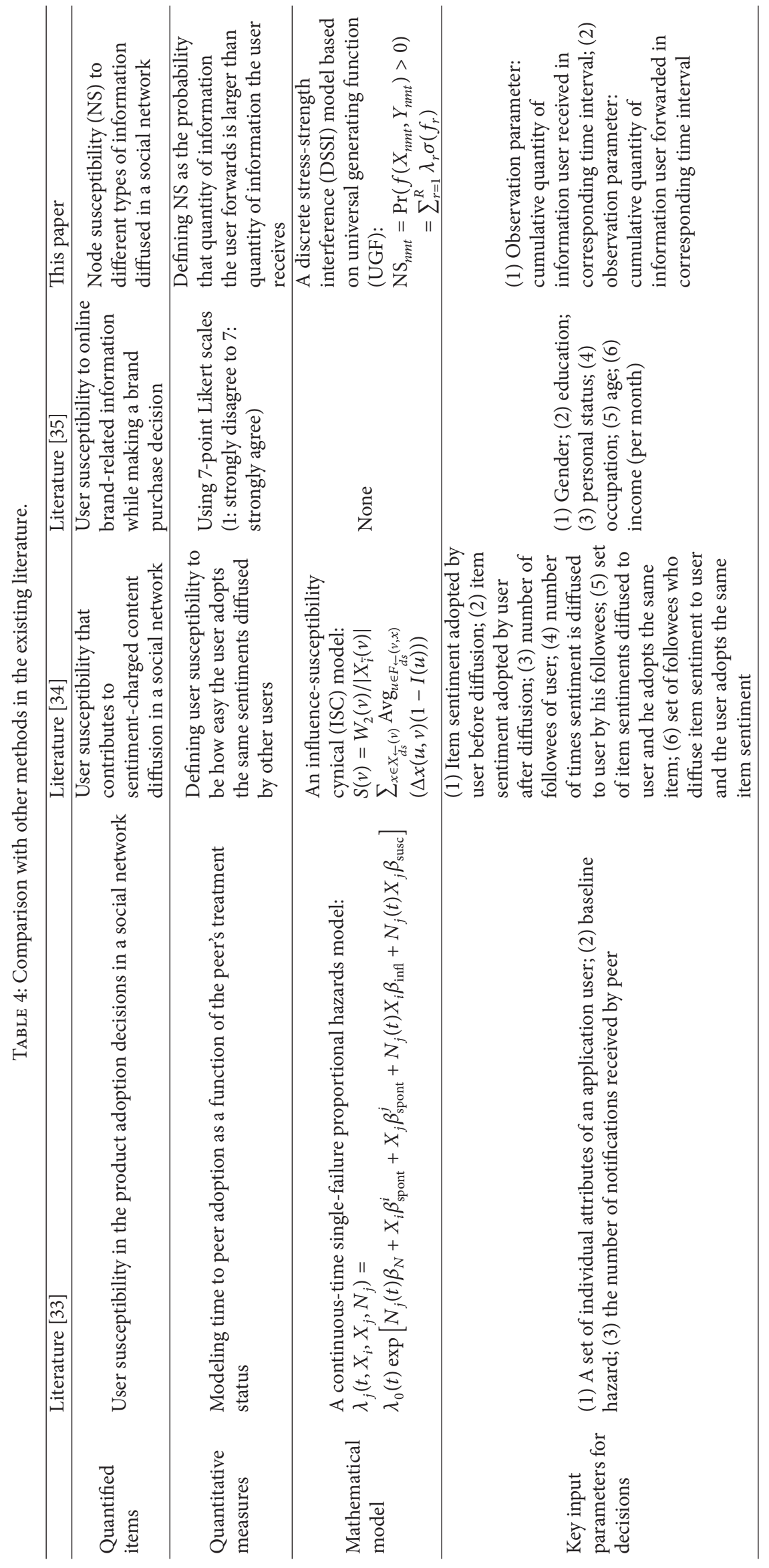




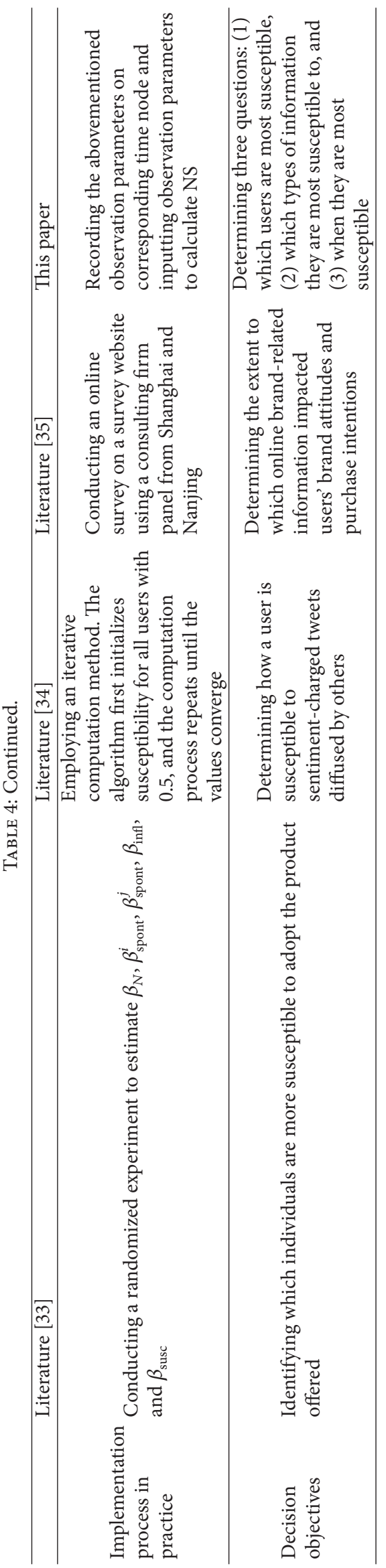


It can be seen from Table 4 that, on the one hand, mathematical models are common tools of quantifying user susceptibility for information diffusion and, on the other hand, given the difference in decision objectives and quantitative measures, the expression of the model is different, making the input parameters for decisions different in different methods. Generally, in the case that the complexities of the models are equivalent, the model with fewer input parameters is easier for decision-makers (practitioners) to implement in practice. Solving the model with more input parameters requires decision-makers to know specialized knowledge and make subjective judgments, which can enhance difficulty and subjectivity of decision-making. In this sense, the models in literature [33] and this paper may be superior to those in literature [34].

With respect to the models in literature [33] and this paper, it is difficult to say which one is better, since different models serve different decision objectives. By conducting a randomized experiment, the decision results in literature [33] are that younger users are more susceptible than older users, and married individuals are the least susceptible in the decision to adopt the product offered. These decision results give some suggestions in the spread of the product in social networks from a macroscopic perspective. In comparison, the decision results (answers to the three questions) in this paper can provide the practitioners with specific reference to make rational decisions on effective information diffusion. In addition, the decision results in literature [33] are static to some extent. Our decision results are dynamic and can be updated with the updated observation data, which can help the practitioners to make information diffusion strategy dynamically.

\section{Conclusion}

In this work, a novel and efficient model for analyzing the effect of nodes on information diffusion is proposed based on universal generating function (UGF) method and discrete stress-strength interference (DSSI) theory. In this model, the effect of user on information diffusion is quantified as node susceptibility (NS), and based on NS the proposed model can help decision-makers to identify which users are most susceptible to the corresponding information at different periods. The contributions of the research can be summarized as follows.

(1) To take into account the influence of randomness and uncertainty, the model introduces a novel and universal evaluation criterion-node susceptibility (NS), based on discrete stress-strength interference (DSSI) theory. Since the calculation of NS is based on the realistic observations of the corresponding random variables, the decision results are rational and objective.

(2) By modeling random forwarding actions, the effect of network nodes on information diffusion is analyzed quantitatively and dynamically. In proposed model, three main questions are solved: (i) which nodes are most susceptible, (ii) which types of information they are most susceptible to, and (iii) when they are most susceptible. The solutions of these questions are very helpful for the practitioners to make rational decisions on effective information diffusion.

(3) Different from the existing related works that mainly focus on extracting the most influential nodes, this work focuses on extracting the most susceptible nodes, which exploits a new idea for studying information diffusion over social networks.

Despite the contributions, this study has several limitations. Although the proposed model can provide objective and dynamic decisions based on NS, it cannot provide the cause of the fluctuation of NS. In other words, decisionmakers may not know why some users are not susceptible and why some types of information are not attractive. In addition, users' forwarding actions may be influenced by network structure or topology relationship of users, which are not considered in the model.

Based on these considerations, this research suggests two avenues for future research. (i) To make the factors that influence the fluctuation of NS more obvious, future researchers can introduce information evaluation mechanism in the model. (ii) When modeling random forwarding actions, network structure or topology relationship of users can be considered as one of the factors influencing information diffusion. It is envisioned to be possible to apply the point-set topology theory and graph theory to address this new issue.

\section{Appendix}

\section{Steps of Calculation of NS by Using SPSS}

The steps of calculation of NS by using SPSS will be given in detail as follows.

Step $1^{*}$ (deriving $X_{n m t}$ 's p.m.f. and $Y_{n m t}$ 's p.m.f. by using SPSS)

(a) Creating the Data Files of $X_{n m t}$ 's Observations and $Y_{n m t}$ 's Observations. In "Variable View," " $X_{n m t}$ " is set as the variable name (Figure 8). Then, $W$ (the number of $X_{n m t}$ 's observations) values of data are imported in "Data View" (Figure 9), and a data file with $W$ rows and one column is created.

Similarly, " $Y_{n m t}$ " is set as another variable name in "Variable View." $Z$ (the number of $Y_{n m t}$ 's observations) values of data are imported in "Data View," and another data file with $Z$ rows and one column is created.

(b) Plotting Histograms with Relative Frequency as Ordinate. We choose the options of "Graphs," "Legacy Dialogs," "Interactive," and "Histogram" in sequence (Figure 10), and then a "Create Histogram" dialog box will appear. In "Assign Variables" list box, we select " $X_{n m t}$ " and drag it into "Create Histogram" dialog box. To set relative frequency as ordinate, we need to select "Percent" in "Assign Variables" list box, and drag it into "Count" dialog box (Figure 11).

In "Histogram" option, we choose "Normal curve" check box, which means that normal curve will appear in the output. Meanwhile, we set "Number of interval" as 7 (decisionmaker can set it as other values as needed), which means that 


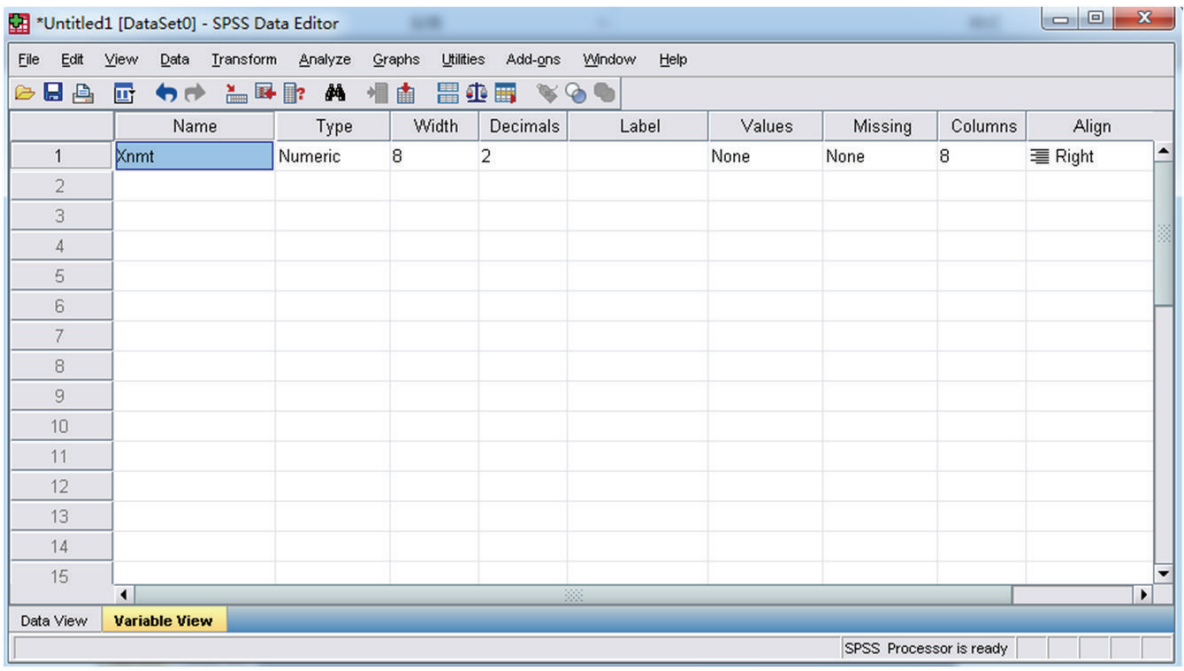

Figure 8: Set $X_{n m t}$ as the variable name in "Variable View."

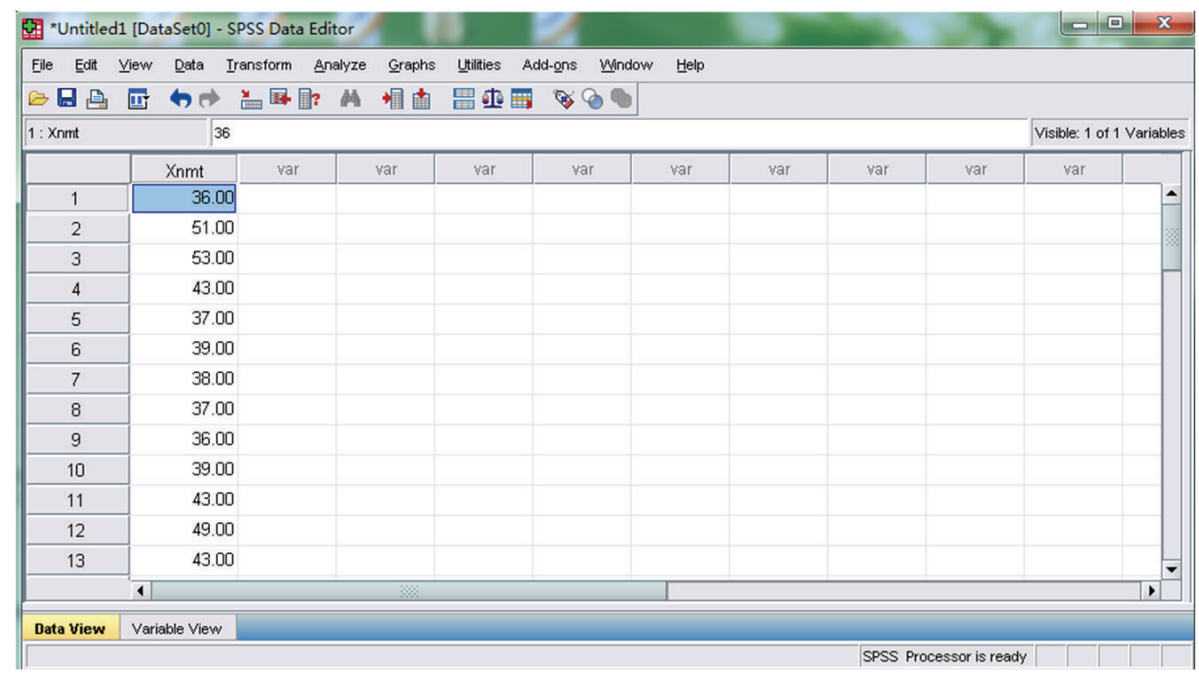

Figure 9: Import values of data in "Data View."

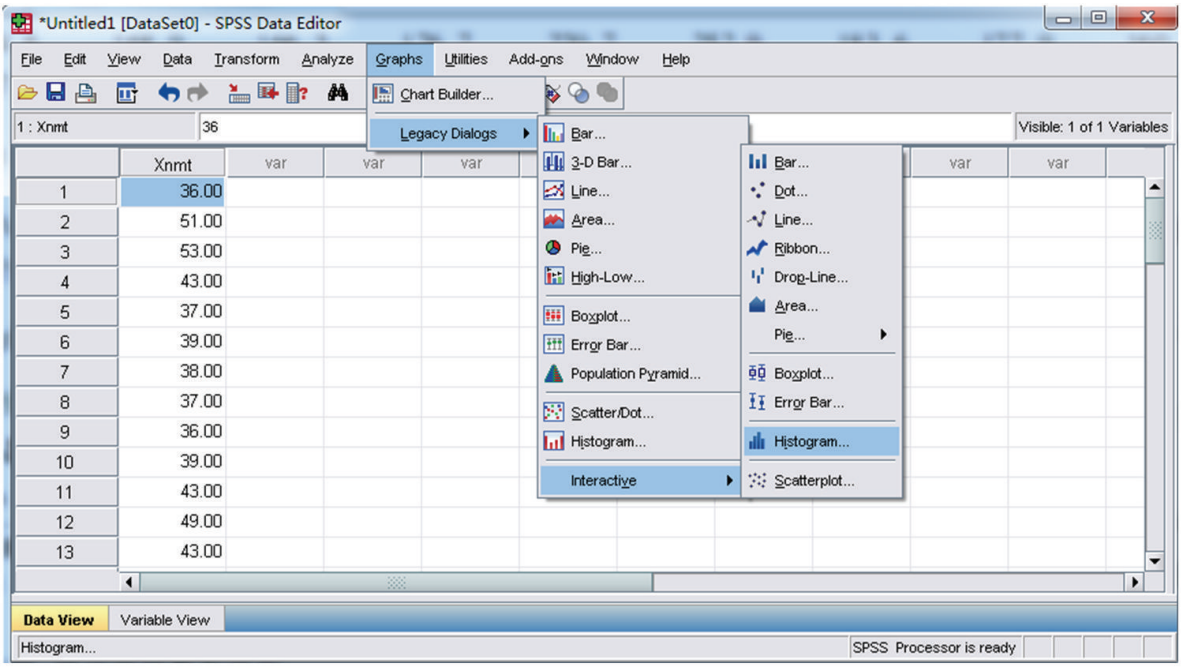

FIGURE 10: “Data Editor” dialog box. 

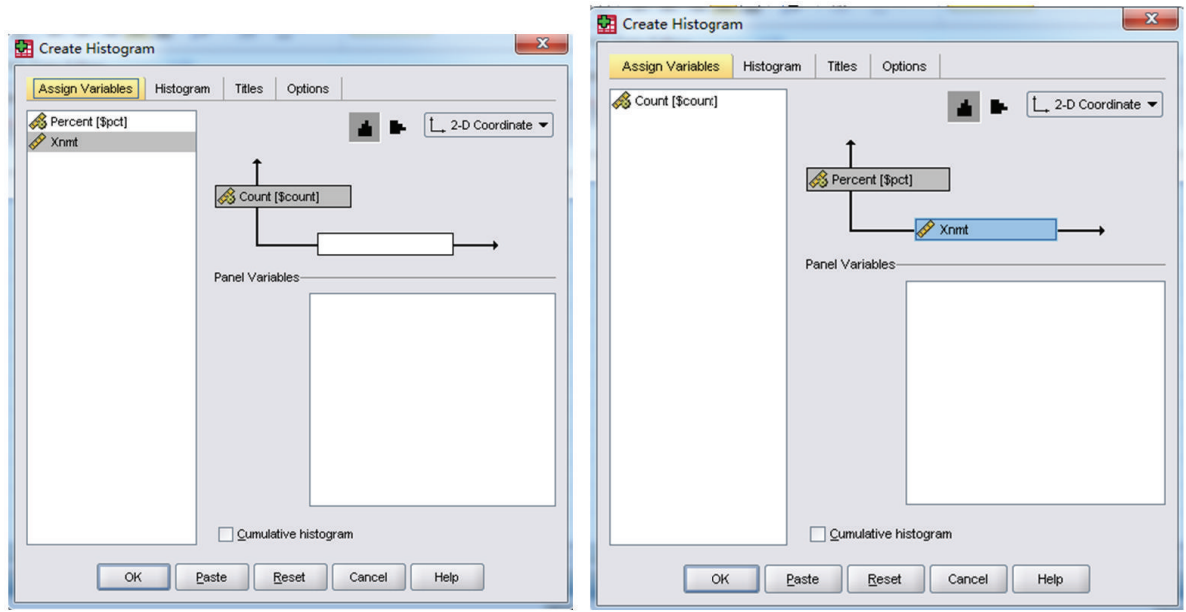

FIGURE 11: "Create Histogram” dialog box.
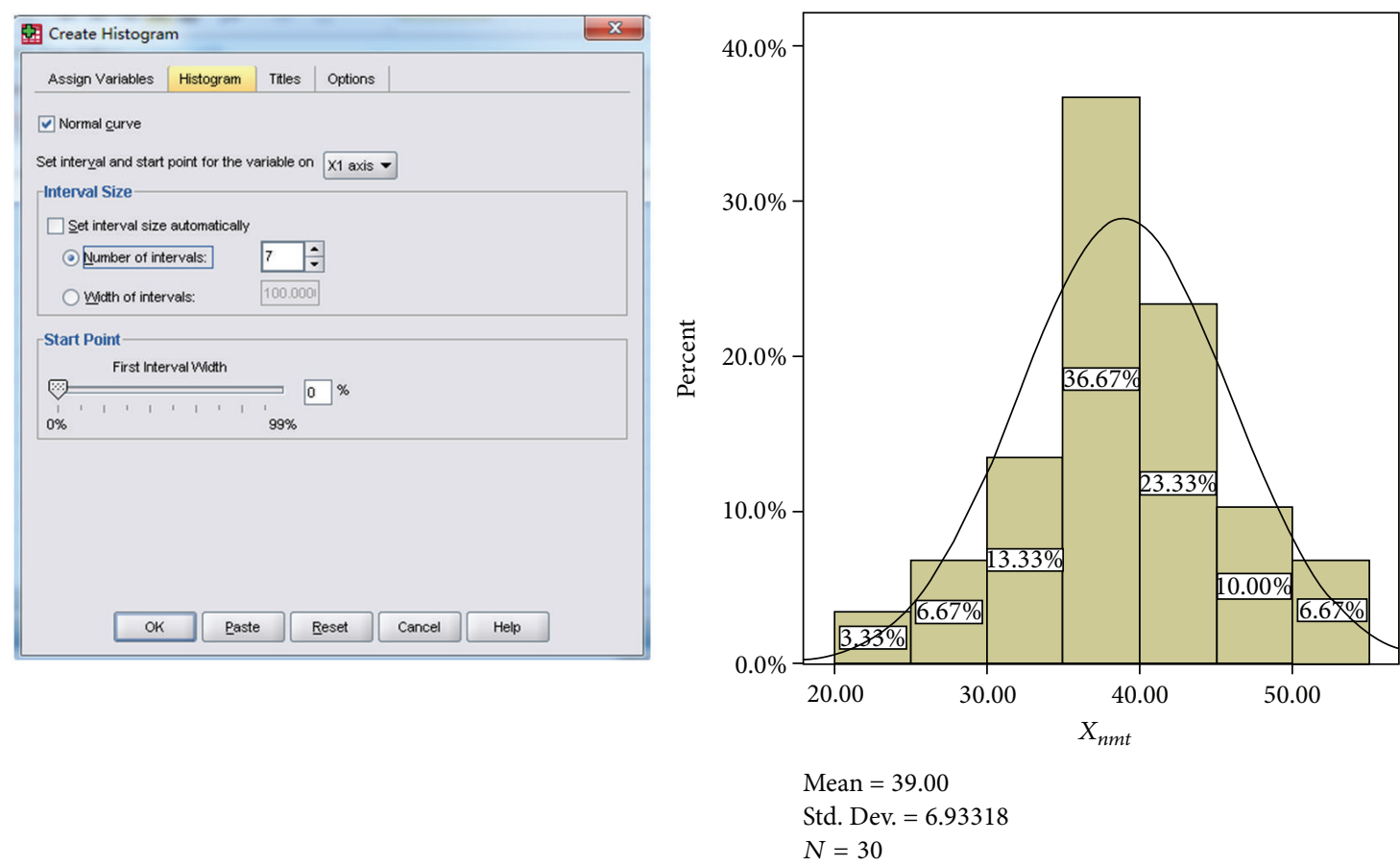

FIGURE 12: Creating histogram with relative frequency as ordinate.

the number of interval of output is 7. Finally, click OK, and the histogram of $X_{n m t}$ 's observations is obtained (Figure 12). Similarly, the histogram of $Y_{n m t}$ 's observations can also be obtained based on the steps above. The general forms of two histograms are shown in Figure 3.

(c) Obtaining $X_{n m t}$ 's p.m.f. and $Y_{n m t}$ 's p.m.f. Based on the Histograms in (b). The midpoint values of each class interval of two histograms are, respectively, treated as possible values of $X_{n m t}$ and $Y_{n m t}$, and relative frequencies of each class interval are treated as corresponding probabilities. Thus, $X_{n m t}$ 's p.m.f. and $Y_{n m t}$ 's p.m.f. can, respectively, be obtained according to (13)-(14) in Section 3.2.2.
Steps $2^{*}$ and $3^{*}$ are the same as Steps 2 and 3 we presented in Section 3.2.2, so we will not repeat here for reasons of brevity.

\section{Notations}

$n$ : Index of users

$m$ : Index of types of information

$t$ : Index of periods

$t j$ : Index of time nodes of the $t$ th period

$U_{n}$ : The $n$th user

$I_{m}$ : The $m$ th type of information

$P_{t}$ : The $t$ th period 


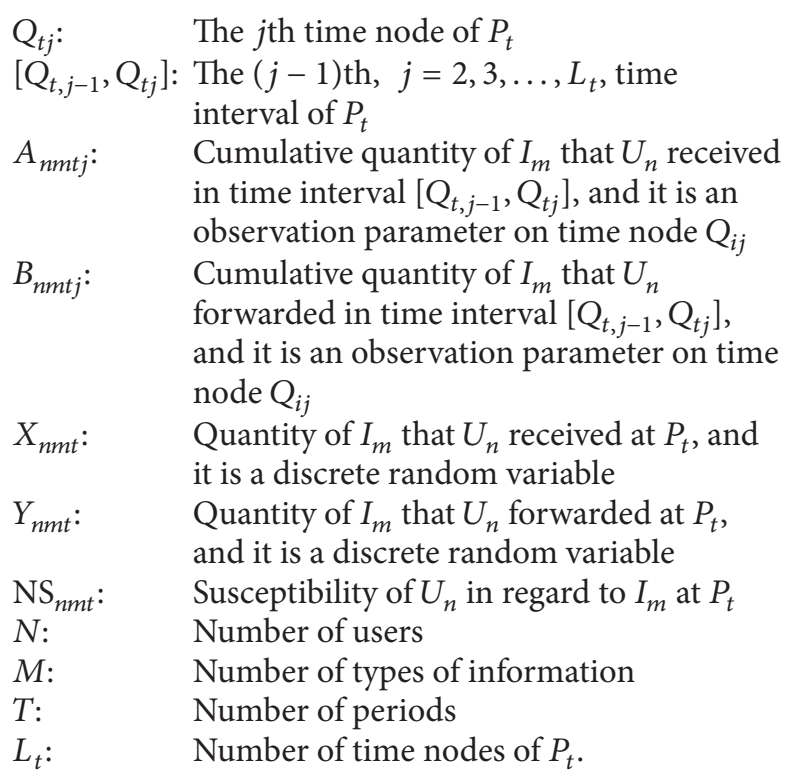

\section{Competing Interests}

The authors declare that they have no competing interests.

\section{Acknowledgments}

This work was supported by the National Natural Science Foundation of China under Grant no. 61173015, no. 61573257, no. 61273046 , and no. 11401273 . The authors should show their great thanks to all of their hard working fellows in the projects above.

\section{References}

[1] E. Bakshy, I. Rosenn, C. Marlow, and L. Adamic, "The role of social networks in information diffusion," in Proceedings of the 21st Annual Conference on World Wide Web (WWW '12), pp. 519-528, Lyon, France, April 2012.

[2] J. Xu, J. X. Xiang, X. Chen, F. B. Liu, and J. J. Yu, "ODMBP: behavior forwarding for multiple property destinations in mobile social networks," Mobile Information Systems, vol. 2016, Article ID 7908328, 11 pages, 2016.

[3] M. Kimura and K. Saito, "Tractable models for information diffusion in social networks," in Knowledge Discovery in Databases: PKDD 2006, vol. 4213 of Lecture Notes in Computer Science, pp. 259-271, Springer, Berlin, Germany, 2006.

[4] Y. Wang, C. Zhao, Q. Xu, Z. Zheng, Z. Chen, and Z. Liu, "Fair secure computation with reputation assumptions in the mobile social networks," Mobile Information Systems, vol. 2015, Article ID 637458, 8 pages, 2015.

[5] Wikipedia, Mobile Social Network, https://en.wikipedia.org/ wiki/Mobile_social_network.

[6] A. L. Traud, P. J. Mucha, and M. A. Porter, "Social structure of facebook networks," Physica A: Statistical Mechanics and Its Applications, vol. 391, no. 16, pp. 4165-4180, 2012.

[7] R. Xiang, J. Neville, and M. Rogati, "Modeling relationship strength in online social networks," in Proceedings of the 19th International Conference on World Wide Web, pp. 981-990, ACM, April 2010.
[8] C. Jiang, Y. Chen, and K. J. R. Liu, "Evolutionary dynamics of information diffusion over social networks," IEEE Transactions on Signal Processing, vol. 62, no. 17, pp. 4573-4586, 2014.

[9] O. Yağan, D. Qian, J. Zhang, and D. Cochran, "Conjoining speeds up information diffusion in overlaying social-physical networks," IEEE Journal on Selected Areas in Communications, vol. 31, no. 6, pp. 1038-1048, 2013.

[10] E. K. Lua, R. Chen, and Z. Cai, "Social trust and reputation in online social networks," in Proceedings of IEEE 17th International Conference on Parallel and Distributed Systems, pp. 811816, Tainan, Taiwan, December 2011.

[11] Wikipedia, Facebook, https://en.wikipedia.org/wiki/Facebook.

[12] G. Miritello, E. Moro, and R. Lara, "Dynamical strength of social ties in information spreading," Physical Review E, vol. 83, no. 4, Article ID 045102, 2011.

[13] C. Jiang, Y. Chen, and K. J. R. Liu, "Graphical evolutionary game for information diffusion over social networks," IEEE Journal on Selected Topics in Signal Processing, vol. 8, no. 4, pp. 524-536, 2014.

[14] J. Jiang, S. Wen, S. Yu, Y. Xiang, and W. Zhou, "K-center: an approach on the multi-source identification of information diffusion," IEEE Transactions on Information Forensics and Security, vol. 10, no. 12, pp. 2616-2626, 2015.

[15] R. Pastor-Satorras and A. Vespignani, "Epidemic spreading in scale-free networks," Physical Review Letters, vol. 86, no. 14, pp. 3200-3203, 2001.

[16] D. Gruhl, R. Guha, D. Liben-Nowell, and A. Tomkins, "Information diffusion through blogspace," in Proceedings of the 13th International World Wide Web Conference (WWW'04), pp. 491501, New York, NY, USA, May 2004.

[17] A.-L. Barabási and R. Albert, "Emergence of scaling in random networks," Science, vol. 286, no. 5439, pp. 509-512, 1999.

[18] L. Donetti, P. I. Hurtado, and M. A. Mũoz, "Network synchronization: optimal and pessimal scale-free topologies," Journal of Physics A: Mathematical and Theoretical, vol. 41, no. 22, Article ID 224008, 2008.

[19] M. Lin and N. Li, "Scale-free network provides an optimal pattern for knowledge transfer," Physica A: Statistical Mechanics and Its Applications, vol. 389, no. 3, pp. 473-480, 2010.

[20] Y. Li, M. Qian, D. Jin, P. Hui, and A. V. Vasilakos, "Revealing the efficiency of information diffusion in online social networks of microblog," Information Sciences, vol. 293, pp. 383-389, 2015.

[21] S. J. Park, Y. S. Lim, and H. W. Park, "Comparing Twitter and YouTube networks in information diffusion: the case of the 'Occupy Wall Street' movement,' Technological Forecasting and Social Change, vol. 95, pp. 208-217, 2015.

[22] M. Kimura, K. Saito, R. Nakano, and H. Motoda, "Extracting influential nodes on a social network for information diffusion," Data Mining and Knowledge Discovery, vol. 20, no. 1, pp. 70-97, 2010.

[23] W. Yu, G. Cong, G. Song, and K. Xie, "Community-based Greedy algorithm for mining top-K influential nodes in mobile social networks," in Proceedings of the 16th ACM SIGKDD International Conference on Knowledge Discovery and Data Mining (KDD '10), pp. 1039-1048, July 2010.

[24] M. U. Ilyas, M. Z. Shafiq, A. X. Liu, and H. Radha, "A distributed and privacy preserving algorithm for identifying information hubs in social networks," in Proceedings of the IEEE International Conference on Computer Communications (IEEE INFOCOM '11), pp. 561-565, Shanghai, China, April 2011. 
[25] J. Yang and S. Counts, "Predicting the speed, scale, range of information diffusion in twitter," in Proceedings of the 4th International AAAI Conference on Weblogs and Social Media, pp. 355-358, 2010.

[26] J. Yang and J. Leskovec, "Modeling information diffusion in implicit networks," in Proceedings of the IEEE International Conference on Data Mining, pp. 599-608, IEEE, Sydney, Australia, December 2010.

[27] K. Saito, M. Kimura, K. Ohara, and H. Motoda, "Super mediator-a new centrality measure of node importance for information diffusion over social network," Information Sciences, vol. 329, pp. 985-1000, 2016.

[28] V. Belák, A. Mashhadi, A. Sala, and D. Morrison, "Phantom cascades: the effect of hidden nodes on information diffusion," Computer Communications, vol. 73, pp. 12-21, 2016.

[29] I. A. Ushakov, "A universal generating function," Soviet Journal of Computer and Systems Sciences, vol. 24, no. 5, pp. 118-129, 1986.

[30] A. Lisnianski and G. Levitin, Multi-State System Reliability: Assessment, Optimization and Applications, World Scientific, Singapore, 2003.

[31] G. Levitin, The Universal Generating Function in Reliability Analysis and Optimization, Springer, London, UK, 2005.

[32] C. Ebeling, An Introduction to Reliability and Maintainability Engineering, Waveland Press, Long Grove, Ill, USA, 2005.

[33] S. Aral and D. Walker, "Identifying influential and susceptible members of social networks," Science, vol. 337, no. 6092, pp. 337341, 2012.

[34] R. K. Lee and E. Lim, "Measuring user influence, susceptibility and cynicalness in sentiment diffusion," in Advances in Information Retrieval: 37th European Conference on IR Research, ECIR 2015, Vienna, Austria, March 29-April 2, 2015. Proceedings, vol. 9022 of Lecture Notes in Computer Science, pp. 411-422, Springer, Berlin, Germany, 2015.

[35] J. Chen, L. Teng, Y. Yu, and X. Yu, "The effect of online information sources on purchase intentions between consumers with high and low susceptibility to informational influence," Journal of Business Research, vol. 69, pp. 467-475, 2016.

[36] Wikipedia, Meituan, https://en.wikipedia.org/wiki/Meituan.com.

[37] baike.baidu, Meituan, http://baike.baidu.com/view/3318024.htm. 

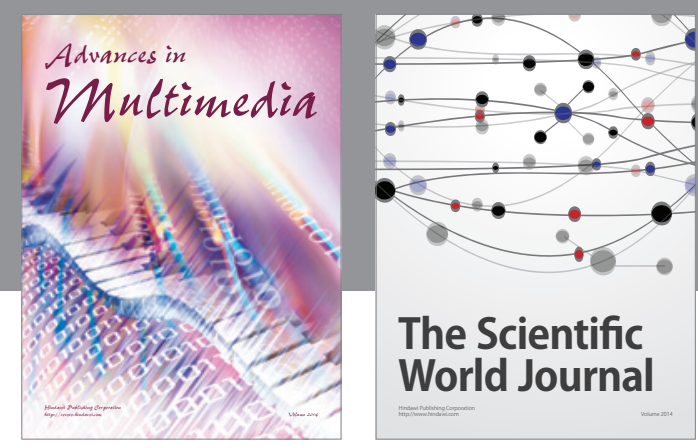

The Scientific World Journal
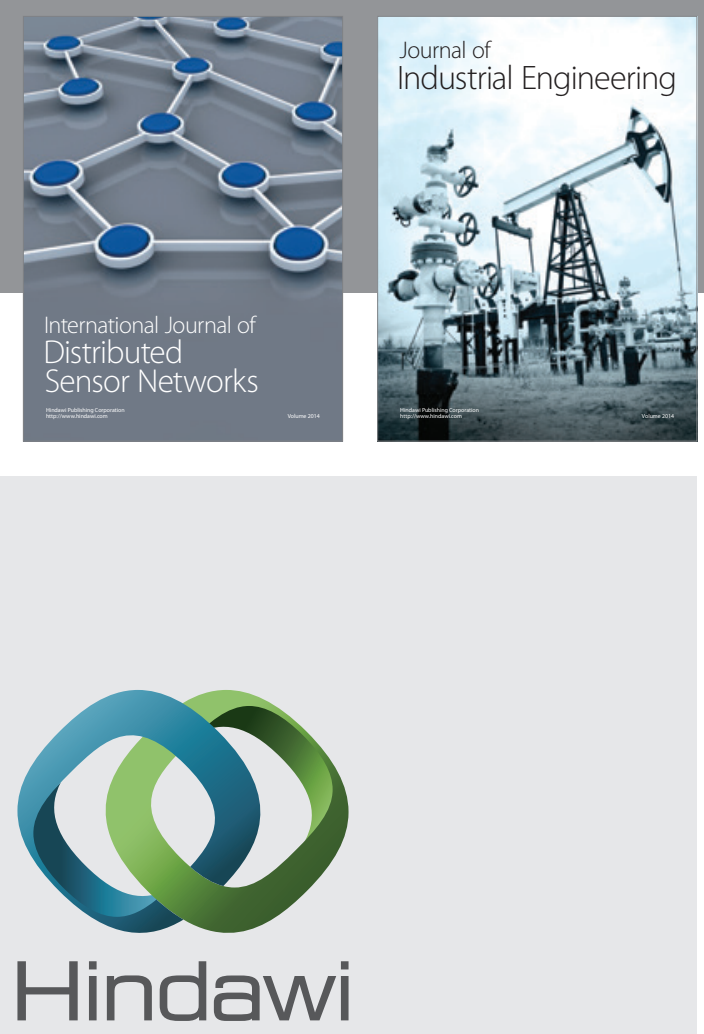

Submit your manuscripts at

http://www.hindawi.com

\section{Computer Networks} and Communications
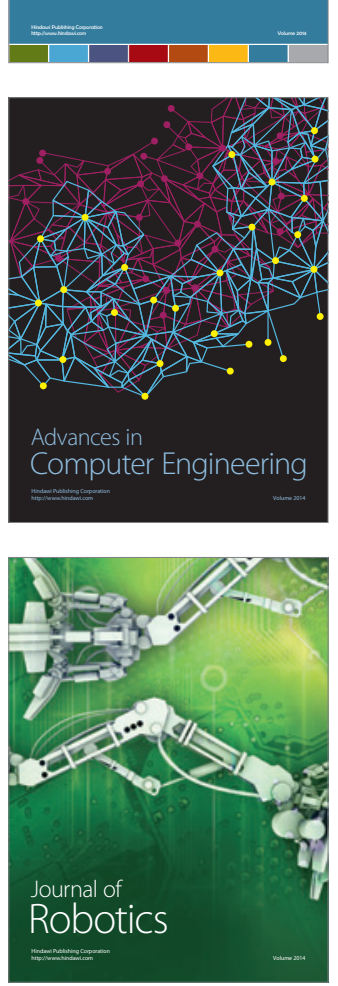
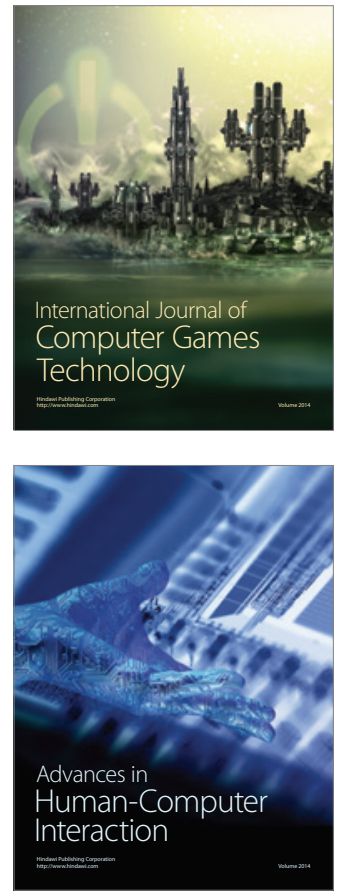
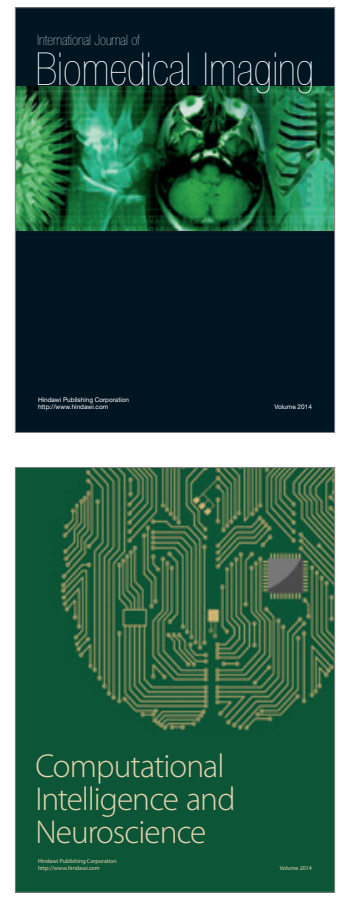
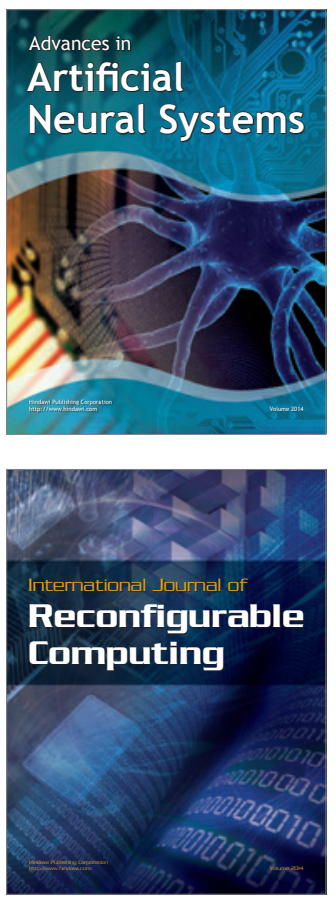
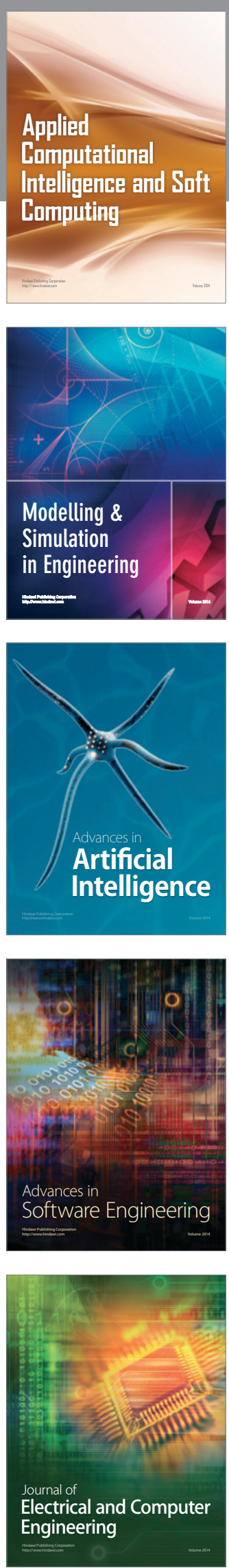